Mon. Not. R. Astron. Soc. 000,118(2012) Printed 21 August $2018 \quad$ (MN LATEX style file v2.2)

\title{
Complex, Quiescent Kinematics in a Highly Filamentary Infrared Dark Cloud ${ }^{\star}$
}

\author{
J. D. Henshaw ${ }^{1} \dagger$, P. Caselli ${ }^{1}$, F. Fontani ${ }^{2}$, I. Jiménez-Serra ${ }^{3}$, J. C. Tan ${ }^{4}$ and \\ A. K. Hernandez \\ ${ }^{1}$ School of Physics and Astronomy, University of Leeds, Leeds LS2 9JT, UK \\ ${ }^{2}$ INAF-Osservatorio Astrofisico di Arcetri, L.go E. Fermi 5, Firenze I-50125, Italy \\ ${ }^{3}$ Harvard-Smithsonian Center for Astrophysics, 60 Garden St., 02138 Cambridge, MA, USA \\ ${ }^{4}$ Department of Astronomy, University of Florida, Gainesville, FL 32611, USA \\ ${ }^{5}$ Department of Astronomy, University of Wisconsin-Madison, 475 N. Charter Street Madison, WI 53706-1582, USA
}

Accepted 2012 October 24. Received 2012 October 24; in original form 2012 June 29

\begin{abstract}
Infrared Dark Clouds (IRDCs) host the initial conditions under which massive stars and stellar clusters form. It is therefore important to study the kinematics, as well as the physical and chemical properties of these regions. Their complex structure however posits challenges in the data interpretation. We have obtained high sensitivity and high spectral resolution observations with the IRAM $30 \mathrm{~m}$ antenna, which allowed us to perform detailed analysis of the kinematics within one IRDC, G035.39-00.33. This cloud has been selected for its highly filamentary morphology and the presence of extended quiescent regions, characteristics of dynamical youth. We focus on the $J=$ $1 \rightarrow 0$ and $J=3 \rightarrow 2$ transitions of $\mathrm{N}_{2} \mathrm{H}^{+}, \mathrm{C}^{18} \mathrm{O}(1-0)$, and make comparison with $\mathrm{SiO}(2-1)$ observations and extinction mapping. Three interacting filaments of gas are found. We report large-scale velocity coherence throughout the cloud, evidenced through small velocity gradients and relatively narrow line widths. This suggests that the merging of these filaments is somewhat "gentle", possibly regulated by magnetic fields. This merging of filaments may be responsible for the weak parsec-scale $\mathrm{SiO}$ emission detected by Jiménez-Serra et al. (2010), via grain mantle vaporization. A systematic velocity shift between the $\mathrm{N}_{2} \mathrm{H}^{+}(1-0)$ and $\mathrm{C}^{18} \mathrm{O}(1-0)$ gas throughout the cloud of $0.18 \pm 0.04 \mathrm{~km} \mathrm{~s}^{-1}$ is also found, consistent with a scenario of collisions between filaments which is still ongoing. The $\mathrm{N}_{2} \mathrm{H}^{+}(1-0)$ is extended throughout the IRDC and it does not only trace dense cores, as found in nearby low-mass star-forming regions. The average $\mathrm{H}_{2}$ number density across the IRDC is $\simeq 5 \times 10^{4} \mathrm{~cm}^{-3}$, at least one order of magnitude larger than in nearby molecular clouds where low-mass stars are forming. A temperature gradient perpendicular to the filament is found. From our study, we conclude that G035.39-00.33 (clearly seen in the extinction map and in $\mathrm{N}_{2} \mathrm{H}^{+}$) has been formed via the collision between two relatively quiescent filaments with average densities of $\simeq 5 \times 10^{3} \mathrm{~cm}^{-3}$, moving with relative velocities of $\simeq 5 \mathrm{~km} \mathrm{~s}^{-1}$. The accumulation of material at the merging points started $\gtrsim 1 \mathrm{Myr}$ ago and it is still ongoing.
\end{abstract}

Key words: stars: formation; ISM: individual objects: G035.39-00.33; ISM: molecules.

\footnotetext{
* Based on observations carried out with the IRAM 30m Telescope. IRAM is supported by INSU/CNRS (France), MPG (Germany) and IGN (Spain).

† E-mail:phy5jh@leeds.ac.uk
}

\section{INTRODUCTION}

In order to disentangle the complex nature of massive (> $8 \mathrm{M}_{\odot}$ ) star and stellar cluster formation, we must first understand the initial conditions under which these objects form. One way to do this is to, firstly, observe dense clouds that have the properties (masses, densities, temperatures etc.) required to produce massive dense cores (hereafter MDCs). 
Henshaw, Caselli, Fontani, Jiménez-Serra, Tan, Hernandez

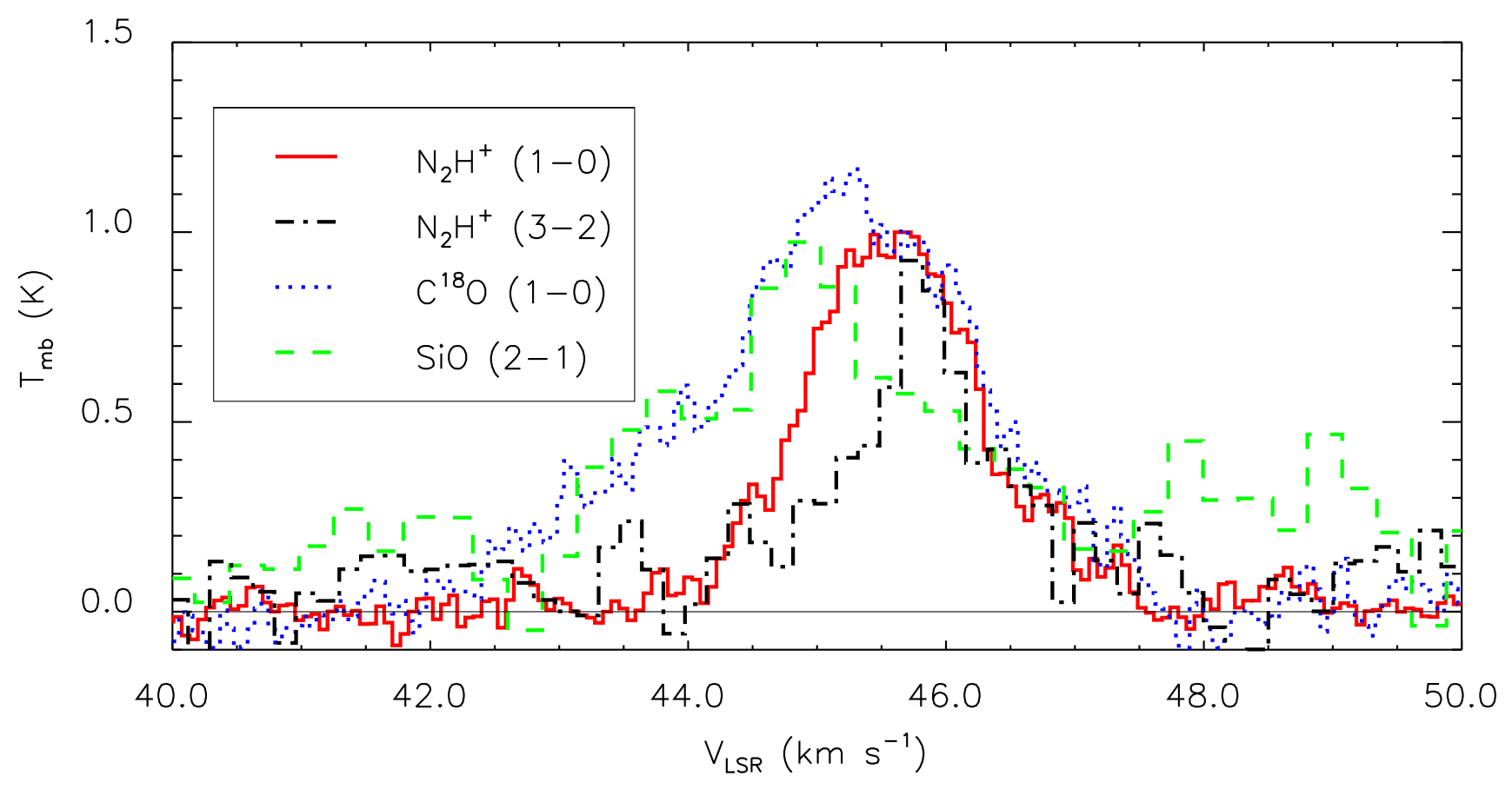

Figure 1. Average spectra of (Red) the isolated component of $\mathrm{N}_{2} \mathrm{H}^{+}(1-0)$, (Black) $\mathrm{N}_{2} \mathrm{H}^{+}(3-2)$, (Blue) $\mathrm{C}^{18} \mathrm{O}(1-0)$, and (Green) $\mathrm{SiO}(2-1)$. The $\mathrm{N}_{2} \mathrm{H}^{+}(1-0),(3-2)$ and $\mathrm{SiO}(2-1)$ intensities have been multiplied by factors of $2,1.5$ and 20 , respectively.

Secondly, it is preferentially required that the MDCs are located sufficiently far away from active star formation sites. This is to avoid complications due to, for instance, feedback effects (such as outflows, stellar winds or UV radiation), and to focus solely on 'pristine' MDCs.

Within the past two decades, one particular group of objects has emerged as an excellent candidate to host the initial conditions of star and stellar cluster formation, Infrared Dark Clouds (hereafter IRDCs). Discovered in the 1990's during mid-IR mapping of the Galaxy with the Infrared Space Observatory (ISO; Perault et al. 1996) and the Midcourse Space Experiment (MSX; Egan et al. 1998), IRDCs are high-extinction clouds, seen in silhouette against the diffuse mid-IR Galactic background. Studies have shown IRDCs are typically cold $(T<25 \mathrm{~K}$; e.g. Pillai et al. 2006, Peretto et al. 2010, Ragan et al. 2011), dense $\left(\mathrm{n}\left(\mathrm{H}_{2}\right)\right.$ $\left.\geqslant 10^{5} \mathrm{~cm}^{-3}\right)$, massive $\left(\sim 10^{2}-10^{5} \mathrm{M}_{\odot}\right)$, have large column densities $\left(\mathrm{N}\left(\mathrm{H}_{2}\right) \geqslant 10^{22} \mathrm{~cm}^{-2}\right.$ ) (e.g. Carev et al. 1998, Rathborne et al. 2006, Simon et al. 2006, Vasyunina et al. 2009), and have large deuterium fractions (e.g. Pillai et al. 2007, Chen et al. 2010). Fontani et al. (2011) found large $\mathrm{N}_{2} \mathrm{D}^{+} / \mathrm{N}_{2} \mathrm{H}^{+}$column density ratios towards a sample of high mass starless cores embedded in cold IRDCs $(\sim 0.3-0.7)$. IRDCs have a range of morphologies, and those having a filamentary structure are thought to be in an early stage of evolution, as described by dynamical formation models of molecular clouds (e.g. van Loo et al. 2007, Hennebelle et al. 2008, Heitsch et al. 2009).

The IRDC studied here, G035.39-00.33, has been selected to (i) have one of the most extreme filamentary structures in the Rathborne et al. (2006) sample of 38 IRDCs studied in millimetre continuum emission, (ii) have extended quiescent regions with relatively low star formation activity, and (iii) be relatively nearby $(\mathrm{D}=2.9 \mathrm{kpc})$. We have focussed specifically on the northern portion of the cloud. The southern portion of the cloud exhibits more tracers of star formation activity ( $8 \mu \mathrm{m}$ and $24 \mu \mathrm{m}$ point sources), and is thought to be in more of an advanced evolutionary stage. The portion over which we have mapped IRDC G035.3900.33 contains three massive cores from the Rathborne et al. (2006) and Butler \& Tan (2009, 2012; hereafter BT09 and BT12 studies). The most massive core (called H6 in BT09 and MM7 in Rathborne et al. 2006) has a mass of $60 \mathrm{M}_{\odot}$ within a radius of $0.15 \mathrm{pc}$ (BT12) and there are no infrared sources at the mass surface density peak. Core H5/MM6 hosts young stellar objects and has a mass of $36 \mathrm{M}_{\odot}$ inside a radius of about $0.13 \mathrm{pc}$, while $\mathrm{H} 4 / \mathrm{MM} 8$ is starless, with a mass of $30 \mathrm{M}_{\odot}$ inside 0.2 pc. Nguven Luong et al. (2011) have also mapped IRDC G035.39-00.33 in the farinfrared with Herschel to investigate the star formation activity within the cloud. They detected a total of 28 cores, 13 of which were considered to be MDCs (with masses > $20 \mathrm{M}_{\odot}$, densities $>2 \times 10^{5} \mathrm{~cm}^{-3}$ ), potentially forming high mass stars. This study was carried out over the full length of the IRDC, and not just toward the northern portion selected here.

Detailed mapping and analysis has recently been carried out towards the northern portion of IRDC G035.3900.33. BT09 and BT12 used extinction mapping in the $8 \mu \mathrm{m}$ Spitzer-IRAC band on a subsample of 10 IRDCs from the Rathborne et al. (2006) sample, providing a high angular resolution $\left(\sim 2^{\prime \prime}\right)$ map of the mass surface density in all 


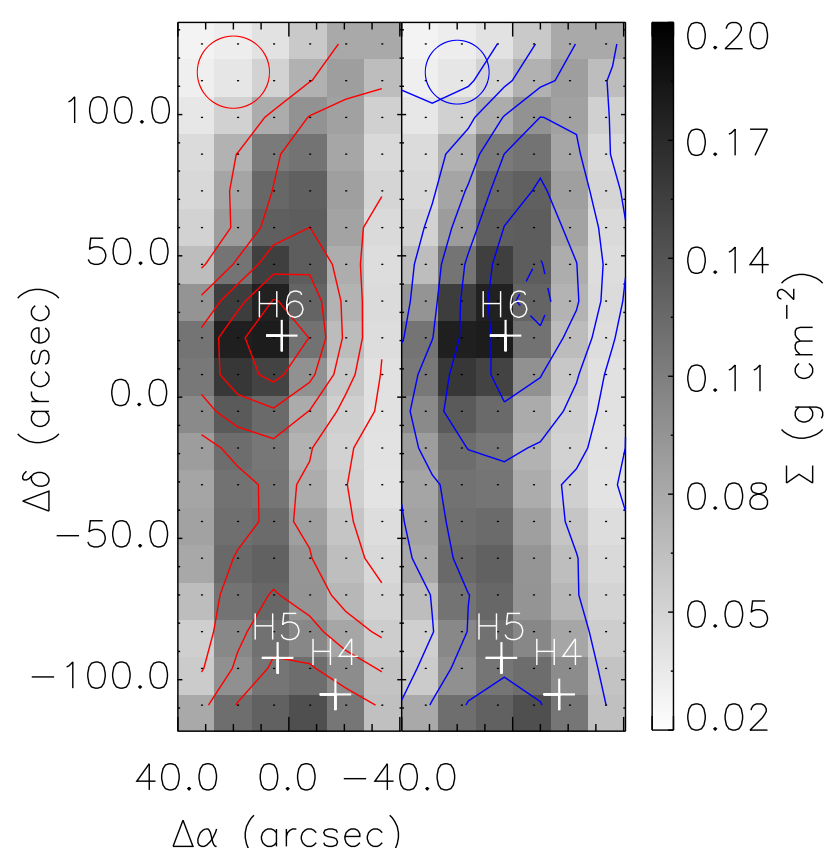

Figure 2. Integrated intensity maps seen in contours, of (Left) $\mathrm{N}_{2} \mathrm{H}^{+}(1-0)$ isolated component and (Right) $\mathrm{C}^{18} \mathrm{O}(1-0)$, overlaid on the mass surface density map smoothed to a $26^{\prime \prime}$ beam. Contours are (Left) $10 \sigma$ to $1.5 \mathrm{~K} \mathrm{~km} \mathrm{~s}^{-1}$, by $5 \sigma$, where $\sigma=0.05 \mathrm{~K}$ $\mathrm{km} \mathrm{s}^{-1}$, (Right) $10 \sigma$ to $4.5 \mathrm{~K} \mathrm{~km} \mathrm{~s}^{-1}$, by $5 \sigma$, where $\sigma=0.15 \mathrm{~K}$ $\mathrm{km} \mathrm{s}^{-1}$. The dashed contour at $5.0 \mathrm{~K} \mathrm{~km} \mathrm{~s}^{-1}$ is added to show the location of the peak. The red and blue circles in the top left of each panel refer to the half-power beam widths of the $\mathrm{N}_{2} \mathrm{H}^{+}(1-0)$, and $\mathrm{C}^{18} \mathrm{O}(1-0)$ maps $\left(26^{\prime \prime}\right.$ and $23^{\prime \prime}$, respectively). White crosses indicate locations of the cores identified by BT12 following their detection in millimetre continuum emission by Rathborne et al. (2006).

cases. Jiménez-Serra et al. 2010) (hereafter, Paper I), presented the first results from a dedicated molecular line study of this IRDC. They found widespread $\mathrm{SiO}(2-1)$ emission covering a large area of the filament. This is suggestive of a large-scale shock caused by the recent collision of molecular filaments, which may have formed the IRDC. Hernandez et al. (2011) (hereafter, Paper II) measured significant levels of CO freeze-out across the IRDC, indicative of cold dense gas. Hernandez \& Tan (2011) studied the dynamics of the IRDC using ${ }^{13} \mathrm{CO}(1-0)$ from the Galactic Ring Survey (Jackson et al. 2006). Their results suggested the possible presence of dynamically important surface pressures around the cloud, perhaps indicating it was relatively young and had not yet settled into virial equilibrium. However, Hernandez et al. (2012) (hereafter, Paper III), revisited this question of virial equilibrium, now using higher resolution $\mathrm{C}^{18} \mathrm{O}$ data and the improved extinction map of BT12. In contrast to Hernandez \& Tan (2011), they found the IRDC exhibited kinematics consistent with models of filamentary virial equilibrium, which requires the cloud to be older than one dynamical time, $\sim 1$ Myr.

In this paper (Paper IV of this series), we focus mainly on $\mathrm{N}_{2} \mathrm{H}^{+}(1-0), \mathrm{N}_{2} \mathrm{H}^{+}(3-2)$ and $\mathrm{C}^{18} \mathrm{O}(1-0) . \mathrm{N}_{2} \mathrm{H}^{+}$ is a known tracer of cold dense regions $\left(>10^{4} \mathrm{~cm}^{-3}\right)$, and

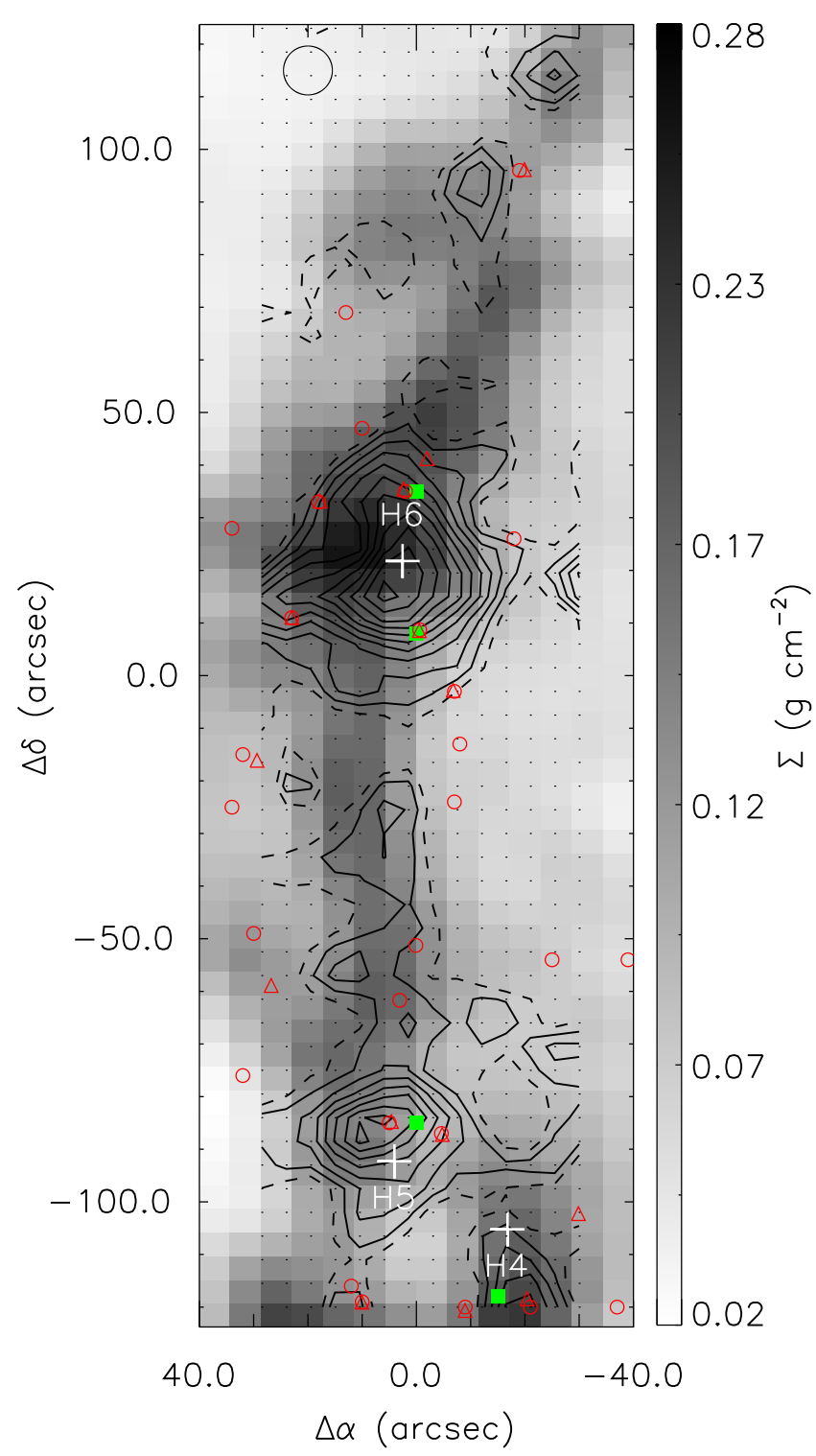

Figure 3. Integrated intensity contours of $\mathrm{N}_{2} \mathrm{H}^{+}(3-2)$, overlaid on the mass surface density map smoothed to a $9^{\prime \prime}$ beam. Contours are $3 \sigma$ to $5.52 \mathrm{~K} \mathrm{~km} \mathrm{~s}^{-1}$, by $1 \sigma$, where $\sigma=0.46 \mathrm{~K} \mathrm{~km} \mathrm{~s}^{-1}$. The dotted contour is the $2 \sigma$ contour. White crosses indicate the positions of the massive cores (BT12). Colored symbols are $4.5 \mu \mathrm{m}$ (green squares), $8 \mu \mathrm{m}$ (red circles) and $24 \mu \mathrm{m}$ (red triangles) sources found in the direction of the IRDC (see Figure 1 of Paper I for more details on these sources).

in low-mass star-forming regions it therefore typically traces the dense cores (e.g. Caselli et al. 2002a). $\mathrm{C}^{18} \mathrm{O}$ is a tracer of more diffuse material $\left(\geqslant 10^{3} \mathrm{~cm}^{-3}\right)$. Due to the fact that it is readily abundant in molecular clouds, $\mathrm{C}^{18} \mathrm{O}$ gives more of an indication of the general morphology of the whole cloud. Unlike ${ }^{12} \mathrm{CO}$ and ${ }^{13} \mathrm{CO}$, the relatively less abundant $\mathrm{C}^{18} \mathrm{O}$ is typically optically thin in molecular clouds, making it an ideal diagnostic tool to study the kinematics of the lower density material. In addition to these tracers we also include the mass surface density derived by BT12 as a tracer of the 
Henshaw, Caselli, Fontani, Jiménez-Serra, Tan, Hernandez

dense material in the filament. The $\mathrm{SiO}(2-1)$ emission, studied extensively in Paper I, is also used to compare our kinematic results, spatially, with the shocked gas within the filament. Finally, the $J=3 \rightarrow 2$ transition of $\mathrm{N}_{2} \mathrm{H}^{+}$is used together with the $J=1 \rightarrow 0$ transition to estimate the number density across the filament.

Details of the observations can be found in Section2 with the results (integrated intensity maps, channel maps, kinematics, column and number density) in Section3. A discussion of our results can be found in Section 4 and conclusions are in Section 5 . Appendix $\mathrm{A}$ outlines the fitting method used to extract information from this data, whereas Appendix B reports tables with the Gaussian and hyperfine structure fits in selected positions across the filament.

\section{OBSERVATIONS}

The $\mathrm{N}_{2} \mathrm{H}^{+}(1-0), \mathrm{N}_{2} \mathrm{H}^{+}(3-2), \mathrm{C}^{18} \mathrm{O}(1-0)$, and $\mathrm{SiO}$ $(2-1)$ observations were mapped with the Instituto de Radioastronomía Milimétrica (IRAM) 30-m telescope at Pico Veleta, Spain. The $\mathrm{N}_{2} \mathrm{H}^{+}$observations were carried out in August 2008 and February 2009, $\mathrm{C}^{18} \mathrm{O}(1-0)$ in August 2008, and $\mathrm{SiO}(2-1)$ observations in December 2008 and February 2009. The large-scale images were obtained with the On-The-Fly (OTF) mapping mode using the offsets $\left(1830^{\prime \prime}, 658^{\prime \prime}\right)$ for the 2008 observations, and $\left(300^{\prime \prime}, 0^{\prime \prime}\right)$ for observations carried out in 2009 (i.e. for part of the $\mathrm{N}_{2} \mathrm{H}^{+}$ and $\mathrm{SiO}$ data, whose emission is concentrated in the IRDC), as off-positions. The central coordinates of the maps are $\alpha(\mathrm{J} 2000)=18^{h} 57^{m} 08^{s}, \delta(\mathrm{J} 2000)=02^{\circ} 10^{\prime} 30^{\prime \prime}\left(l=35^{\circ} .517\right.$, $\left.b=-0^{\circ} .274\right)$. All observations were carried out using the old ABCD receivers. The VErsatile SPectrometer Assembly (VESPA) provided spectral resolutions between 20 and $80 \mathrm{kHz}$. Information on the beam sizes, frequencies and velocity resolutions can all be found in Table1 Typical system temperatures range from 100-200 K for all line observations except for $\mathrm{N}_{2} \mathrm{H}^{+}(3-2)$ which has a value of $\mathrm{T}_{\mathrm{SYS}}$ $\sim 750 \mathrm{~K}$. All intensities were calibrated in units of antenna temperature, $\mathrm{T}_{\mathrm{A}}^{*}$. To convert these intensities into units of main-beam brightness temperature (assuming a unity filling factor), $\mathrm{T}_{\mathrm{MB}}$, we have used the beam and forward efficiencies listed in Table1 Saturn was used to calculate the focus, and pointing was checked every 2 hours on G34.3+0.2. The data were calibrated with the chopper-wheel technique (Kutner \& Ulich 1981), with a calibration uncertainty of $\sim 20 \%$.

As in Paper III, we utilize the $8 \mu \mathrm{m}$ extinction derived mass surface density map of BT12, as modified by Kainulainen \& Tan (2012) (hereafter, KT12) to include corrections for the presence of the NIR extinction derived IRDC envelope. The mass surface density map has 2" resolution, which we have smoothed to 26" (unless otherwise stated), so as the data is of comparable resolution to the $\mathrm{N}_{2} \mathrm{H}^{+}(1-0)$ and $\mathrm{C}^{18} \mathrm{O}(1-0)$ observations.

\section{RESULTS}

\subsection{Spectra and integrated intensities}

The spectra of the isolated $\mathrm{N}_{2} \mathrm{H}^{+}(1-0)$ hyperfine component $\left(\mathrm{F}_{1}, \mathrm{~F}=0,1 \rightarrow 1,2\right)$, the $\mathrm{N}_{2} \mathrm{H}^{+}(3-2)$, the $\mathrm{C}^{18} \mathrm{O}(1-0)$, and the $\mathrm{SiO}(2-1)$, averaged across the whole mapped area, are shown in Figure 1. The spectra are plotted in units of main beam brightness temperature, with the $\mathrm{N}_{2} \mathrm{H}^{+}(1-0)$, $\mathrm{N}_{2} \mathrm{H}^{+}(3-2)$ and $\mathrm{SiO}(2-1)$ being multipled by factors of $2,1.5$ and 20, respectively, for comparison. The four molecular gas tracers have different centroid velocities, with the highest density tracer, $\mathrm{N}_{2} \mathrm{H}^{+}(3-2)$, peaking at the largest velocity, followed by $\mathrm{N}_{2} \mathrm{H}^{+}(1-0), \mathrm{C}^{18} \mathrm{O}(1-0)$ and $\mathrm{SiO}$ $(2-1)$. The $\mathrm{SiO}(2-1)$ shows the broadest width, with emission present up to velocities of about $50 \mathrm{~km} \mathrm{~s}^{-1}$. The $\mathrm{C}^{18} \mathrm{O}$ line encloses the emission of the other species (except for the high velocity emission of the $\mathrm{SiO}(2-1)$ ) and presents an asymmetric profile indicative of multiple velocity components along the line of sight. Although shifts in velocities relative to the $\mathrm{SiO}$ line are expected given that $\mathrm{SiO}$ typically traces shocked material (e.g. Jiménez-Serra et al. 2009), the significant shifts in centroid velocities observed in the more quiescent molecular cloud tracers $\mathrm{C}^{18} \mathrm{O}$ and $\mathrm{N}_{2} \mathrm{H}^{+}$ represent a clear difference from low-mass star-forming regions (Walsh et al. 2004, Hacar \& Tafalla 2011).

Figure 2 presents integrated intensity maps of the $J=$ $1 \rightarrow 0$ transitions of both $\mathrm{N}_{2} \mathrm{H}^{+}$(Red contours; $\mathrm{F}_{1}, \mathrm{~F}=0,1$ $\rightarrow 1,2$ hyperfine component), and $\mathrm{C}^{18} \mathrm{O}$ (Blue contours), convolved with the respective beams (see Table1) and using a pixel size of half the respective beam sizes. In each map, the spectra have been integrated between 40 and $50 \mathrm{~km} \mathrm{~s}^{-1}$, to cover all the emission (see Figure1). The $\mathrm{N}_{2} \mathrm{H}^{+}(1-0)$ and $\mathrm{C}^{18} \mathrm{O}(1-0)$ maps have been superimposed on the mass surface density map smoothed to $26^{\prime \prime}$ angular resolution. Overlaid are white crosses to indicate the location of the three massive cores seen in the millimetre continuum emission (Rathborne et al. 2006) and in the unsmoothed extinction map (Butler \& Tan 2009). The integrated intensity map of the $\mathrm{N}_{2} \mathrm{H}^{+}(3-2)$ emission is shown in Figure 3] overlaid on the mass surface density map smoothed to the IRAM $9^{\prime \prime}$ beam at the frequency of this transition.

From Figures 2 and 3 we find that the $\mathrm{N}_{2} \mathrm{H}^{+}(1-0)$ and $(3-2)$ emitting regions are extended across a large portion of the filament. This is different from low-mass starforming regions, where $\mathrm{N}_{2} \mathrm{H}^{+}$mainly traces the dense cores (Caselli et al. 2002a, André et al. 2007, Friesen et al. 2010), whereas filaments can be seen with lower density tracers such as $\mathrm{C}^{18} \mathrm{O}(1-0)$ (Mizuno et al. 1995, Hacar \& Tafalla 2011). Given that the critical density of the $\mathrm{N}_{2} \mathrm{H}^{+}(1-0)$ transition is $\simeq 10^{5} \mathrm{~cm}^{-3}$, this is a clear indication that the overall number density of this IRDC is similar to the average density of nearby low-mass dense cores (i.e. at least one order of magnitude higher than the average density of the clouds within which nearby low-mass cores are embedded, $\leqslant 10^{3} \mathrm{~cm}^{-3}$; e.g. Pineda et al. 2008). $\mathrm{N}_{2} \mathrm{H}^{+}(3-2)$ peaks towards two out of the three massive cores mentioned above (white crosses in Figure 3). The peak in both transitions of $\mathrm{N}_{2} \mathrm{H}^{+}$is seen to be coincident with the position of core $\mathrm{H} 6$, whereas the $\mathrm{C}^{18} \mathrm{O}(1-0)$ peaks away from this (within $\sim 1$ beam size). This is due to the freeze-out of $\mathrm{CO}$, most prominent towards core $\mathrm{H} 6$ (Paper II). Emission from both $\mathrm{N}_{2} \mathrm{H}^{+}$ transitions traces the morphology of the extinction fairly well, whereas the emission from the $\mathrm{C}^{18} \mathrm{O}(1-0)$ covers a larger area.

Figure 4 shows the total integrated intensities of the $J=1 \rightarrow 0\left(\mathrm{~F}_{1}, \mathrm{~F}=0,1 \rightarrow 1,2\right)$ and $J=3 \rightarrow 2$ transitions of $\mathrm{N}_{2} \mathrm{H}^{+}, \mathrm{C}^{18} \mathrm{O}(1-0)$, and $\mathrm{SiO}(2-1)$ as a function 


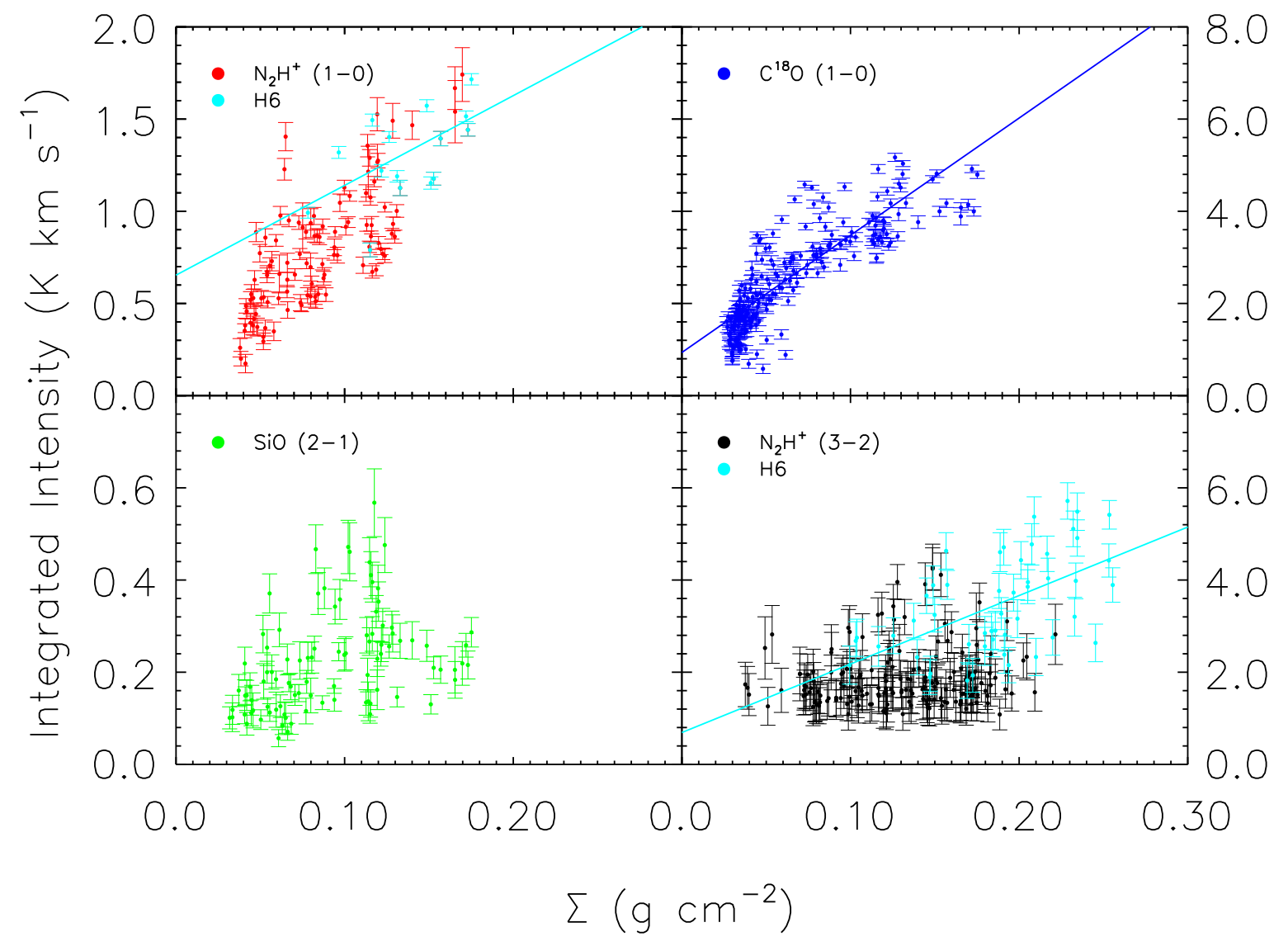

Figure 4. Integrated intensity as a function of mass surface density for the (Red) $\mathrm{N}_{2} \mathrm{H}^{+}(1-0)$ isolated component, (Blue) $\mathrm{C}^{18} \mathrm{O}(1-0)$, (Green) $\mathrm{SiO}(2-1)$ (all smooted to $26^{\prime \prime}$ ), and the $9^{\prime \prime}$ resolution (Black) $\mathrm{N}_{2} \mathrm{H}^{+}(3-2)$. The $\mathrm{N}_{2} \mathrm{H}^{+}$integrated intensity spectra within the starless core $\mathrm{H} 6$ are displayed in Cyan.

of mass surface density. Intensities are integrated over a velocity range of $40-50 \mathrm{~km} \mathrm{~s}^{-1}$. To highlight the properties of the most massive core in the region, $\mathrm{N}_{2} \mathrm{H}^{+}$data from core H6 have different color:1. Significant correlation (regression value, -value $=0.8)$ is found between $\mathrm{N}_{2} \mathrm{H}^{+}(1-0)$ integrated intensity and mass surface density, over the full cloud (including core $\mathrm{H} 6$ ). This is to be expected, as the $\mathrm{N}_{2} \mathrm{H}^{+}$is tracing the dense gas. A significant correlation is also found across core $\mathrm{H} 6(\mathrm{r}$-value $=0.6)$, despite the fewer data points. For the $\mathrm{N}_{2} \mathrm{H}^{+}(3-2)$ we have compared the line integrated intensity and mass surface density, both smoothed to $9^{\prime \prime}$. The correlation for $\mathrm{N}_{2} \mathrm{H}^{+}(3-2)$ is however not as strong $(\mathrm{r}$-value $=0.5)$, over the full cloud, probably due to the fact that this line is not just sensitive to number density but also to temperature (see e.g. Pagani et al. 2007), and warm gas may not be correlated with the mass surface and number density (see Section 3.5). A slightly stronger corre-

1 In order to separate this core from the rest of the data we have taken the $5 \sigma$ level of the $\mathrm{N}_{2} \mathrm{H}^{+}(1-0)$ and $(3-2)$ intensity (between $-20.0 \leqslant \Delta \alpha \leqslant 20.0$ and $-10.0 \leqslant \Delta \delta \leqslant 50.0$ ), and attribute this to the core. lation is present toward core $\mathrm{H} 6$ ( $\mathrm{r}$-value $=0.55)$, where the line is brightest. There is also a strong correlation (r-value $=0.8)$ between the $\mathrm{C}^{18} \mathrm{O}(1-0)$ integrated intensity and the mass surface density. A weaker correlation (r-value = $0.45)$ between the $\mathrm{SiO}(2-1)$ integrated intensity and the mass surface density is observed. This, along with the fact that the centroid velocities of the $\mathrm{SiO}$ and $\mathrm{N}_{2} \mathrm{H}^{+}$transitions are not coincident (see Figure1), suggests that the narrow $\mathrm{SiO}(2-1)$ emission is mainly associated with the lower density gas traced by $\mathrm{C}^{18} \mathrm{O}\left(\mathrm{n}_{H} \sim 10^{3} \mathrm{~cm}^{-3}\right)$, despite the relatively high critical density of the transition $\left(\mathrm{n}_{c r} \sim 10^{5}\right.$ $\left.\mathrm{cm}^{-3}\right)$. Therefore, we expect the narrow SiO $(2-1)$ lines to be subthermally excited, which would explain their low brightness (Paper I).

\subsection{Kinematics}

\subsubsection{Multiple velocity components}

Figure 5 displays the individual $\mathrm{N}_{2} \mathrm{H}^{+}(1-0)\left(\mathrm{F}_{1}, \mathrm{~F}=0,1\right.$ $\rightarrow 1,2)$ and $\mathrm{C}^{18} \mathrm{O}(1-0)$ spectra over the full extent of the region mapped in $\mathrm{N}_{2} \mathrm{H}^{+}(1-0)$. This is overlaid on 


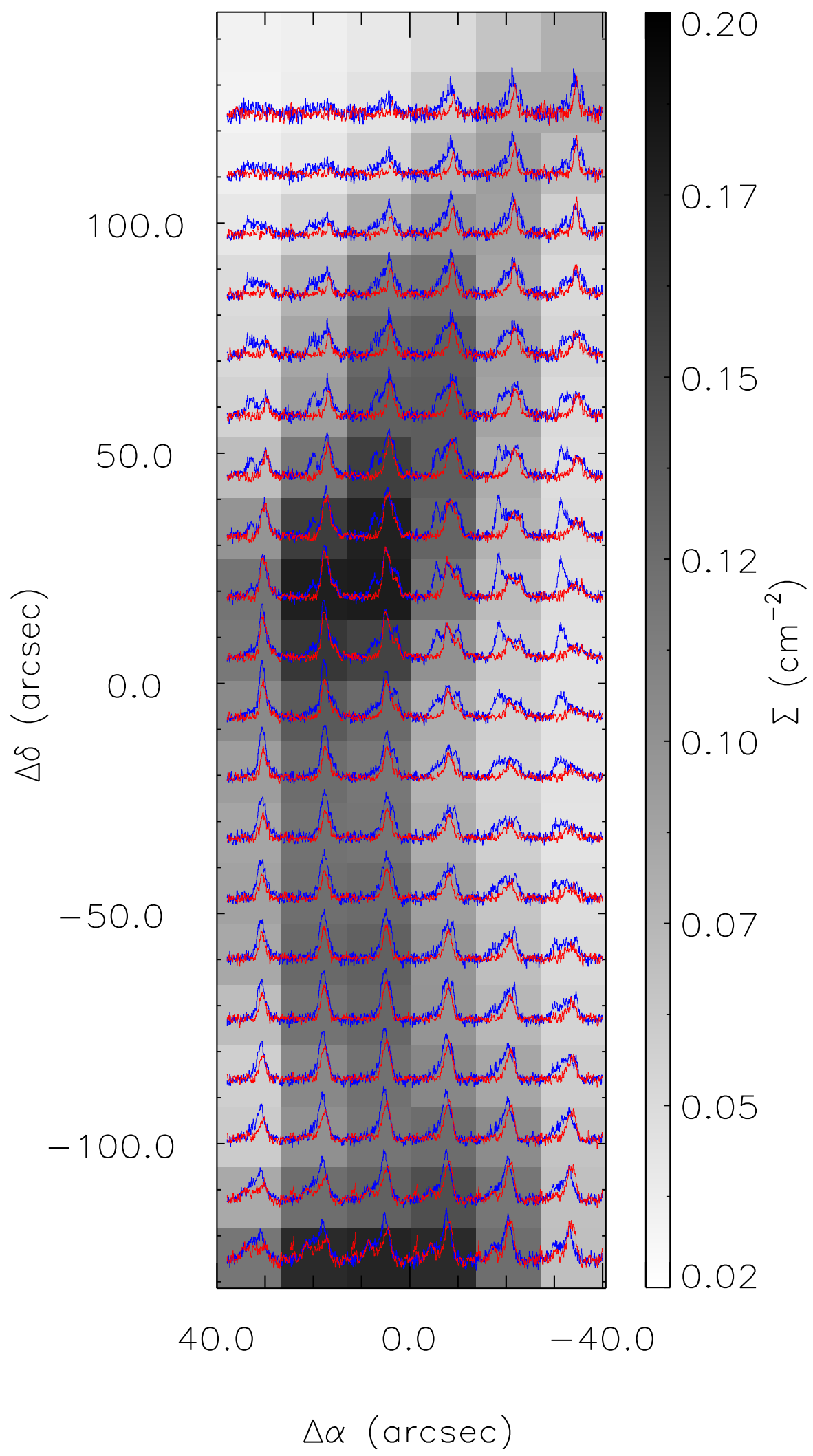

Figure 5. Spectra of the (Red) $\mathrm{N}_{2} \mathrm{H}^{+}(1-0)\left(\mathrm{F}_{1}, \mathrm{~F}=0,1 \rightarrow 1,2\right)$ and (Blue) $\mathrm{C}^{18} \mathrm{O}(1-0)$ at all positions in the cloud, overlaid on the mass surface density map of KT12. Spectra are shown between $41-49 \mathrm{~km} \mathrm{~s}^{-1}$ and $-0.1-1.2 \mathrm{~K}$. The intensity of the $\mathrm{N}_{2} \mathrm{H}^{+}(1-0)$ has been multiplied by a factor of 2 for clarity. The $\mathrm{C}^{18} \mathrm{O}(1-0)$ has been smoothed to $26^{\prime \prime}$, and regridded this to $13^{\prime \prime}$, to make direct comparison with the $\mathrm{N}_{2} \mathrm{H}^{+}(1-0)$ data. 
Table 1. Observed molecular transitions, line frequencies, spectral resolutions, telescope beam sizes, beam correction factors for the ABCD receivers at the IRAM $30 \mathrm{~m}$ telescope.

\begin{tabular}{cccccc}
\hline \hline Transition & $\begin{array}{c}\text { Frequency } \\
(\mathrm{MHz})\end{array}$ & $\begin{array}{c}\text { Spectral } \\
\text { Resolution }\left(\mathrm{km} \mathrm{s}^{-1}\right)\end{array}$ & $\begin{array}{c}\text { Beam Size } \\
(\operatorname{arcsec})\end{array}$ & $\begin{array}{c}\text { Beam } \\
\text { Efficiency }\end{array}$ & $\begin{array}{c}\text { Forward } \\
\text { Efficiency }\end{array}$ \\
\hline $\mathrm{SiO} J=2 \rightarrow 1$ & $86846.9600^{a}$ & $2.68 \times 10^{-1}$ & 28 & 0.77 & 0.98 \\
$\mathrm{~N}_{2} \mathrm{H}^{+} J=1 \rightarrow 0$ & $93173.7637^{b}$ & $6.28 \times 10^{-2}$ & 26 & 0.76 & 0.98 \\
$\mathrm{C}^{18} \mathrm{O} J=1 \rightarrow 0$ & $109782.1730^{c}$ & $5.33 \times 10^{-2}$ & 23 & 0.73 & 0.97 \\
$\mathrm{~N}_{2} \mathrm{H}^{+} J=3 \rightarrow 2$ & $279511.8320^{b}$ & $4.19 \times 10^{-2}$ & 9 & 0.49 & 0.90 \\
\hline
\end{tabular}

$a$ : Jiménez-Serra et al. $\overline{(2010)}$,

$b$ : Pagani et al. (2009),

$c$ : Cazzoli et al. (2003)

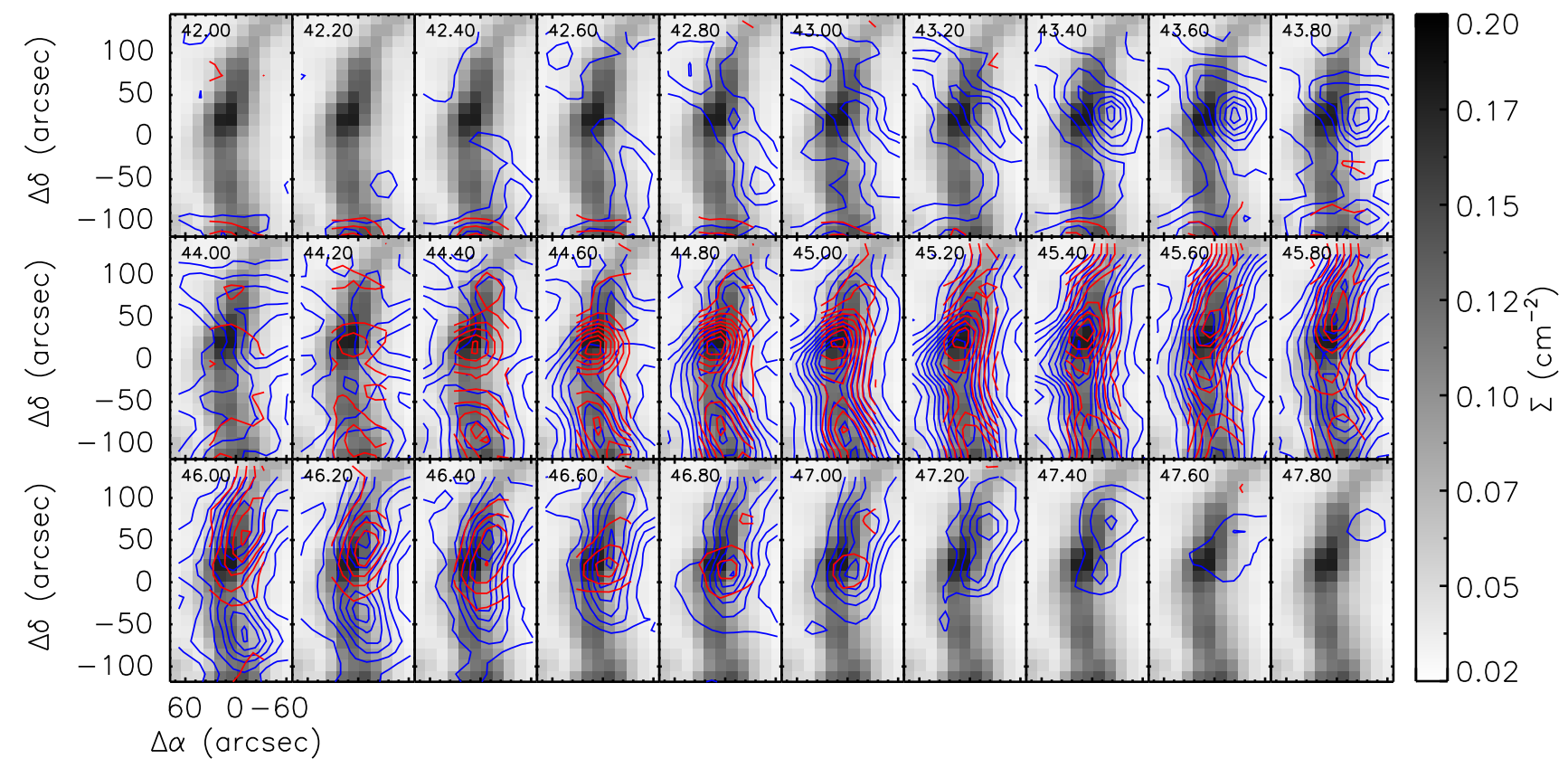

Figure 6. G035.39-00.33 channel maps of (Red) $\mathrm{N}_{2} \mathrm{H}^{+}(1-0)\left(\mathrm{F}_{1}, \mathrm{~F}=0,1 \rightarrow 1,2\right)$ and (Blue) $\mathrm{C}^{18} \mathrm{O}(1-0)$, overlaid on the mass surface density map. Intensity maps integrated from $43 \mathrm{~km} \mathrm{~s}^{-1}$ to $48 \mathrm{~km} \mathrm{~s}^{-1}$ and in steps of $0.2 \mathrm{~km} \mathrm{~s}^{-1}$. Contours for $\mathrm{N}_{2} \mathrm{H}^{+}(1-0)$ in all cases are from $3 \sigma$, increasing in steps of $3 \sigma\left(0.02 \mathrm{~K} \mathrm{~km} \mathrm{~s}^{-1}\right)$. Contours for $\mathrm{C}^{18} \mathrm{O}(1-0)$ in all cases are from $3 \sigma$, increasing in steps of $3 \sigma\left(0.04 \mathrm{~K} \mathrm{~km} \mathrm{~s}^{-1}\right)$.

top of the mass surface density map, smoothed to $26^{\prime \prime}$, to match the angular resolution. As previously noted in Paper I, multiple velocity components appear at various positions within the cloud (e.g. around core H6 and toward the South). This is more apparent in $\mathrm{C}^{18} \mathrm{O}(1-0)$ as it traces the more abundant, less dense gas along the line of sight. However, the line morphologies of the $\mathrm{C}^{18} \mathrm{O}(1-0)$ are echoed in the South and Central positions in $\mathrm{N}_{2} \mathrm{H}^{+}(1-0)$. The fact that the profiles of the $\mathrm{N}_{2} \mathrm{H}^{+}(1-0)$ line are similar to the optically thin $\mathrm{C}^{18} \mathrm{O}(1-0)$, means that we are indeed seeing multiple velocity components along the line of sight and not optical depth effects. In particular, the $\mathrm{N}_{2} \mathrm{H}^{+}(1-0)$ spectra around the peak of core H6 (see offsets $(-7.5,8),(-7.5,20),(5.5,8)$, and (5.5, 21)), with a stronger blue peak typically observed in collapsing low-mass dense cores (e.g. Crapsi et al. 2005), are just the superposition of different velocity components.

A more detailed view of the gas distribution can be found in Figure 6 Here we present the channel maps of the emission seen in $\mathrm{N}_{2} \mathrm{H}^{+}(1-0)\left(\mathrm{F}_{1}, \mathrm{~F}=0,1 \rightarrow 1,2\right)$ and $\mathrm{C}^{18} \mathrm{O}(1-0)$, between $42-48 \mathrm{~km} \mathrm{~s}^{-1}$, in velocity increments of $0.2 \mathrm{~km} \mathrm{~s}^{-1}$, superimposed onto the mass surface density map smoothed to $26^{\prime \prime}$. From the figure, it is evident that the additional components seen in the spectra of Figure 5, have varying morphologies (see also Paper I for a similar analysis using $\mathrm{C}^{18} \mathrm{O}(2-1)$ data). The first of these components (Filament 1) can be seen between $42-44 \mathrm{~km} \mathrm{~s}^{-1}$. In $\mathrm{C}^{18} \mathrm{O}(1-0)$ 
Henshaw, Caselli, Fontani, Jiménez-Serra, Tan, Hernandez

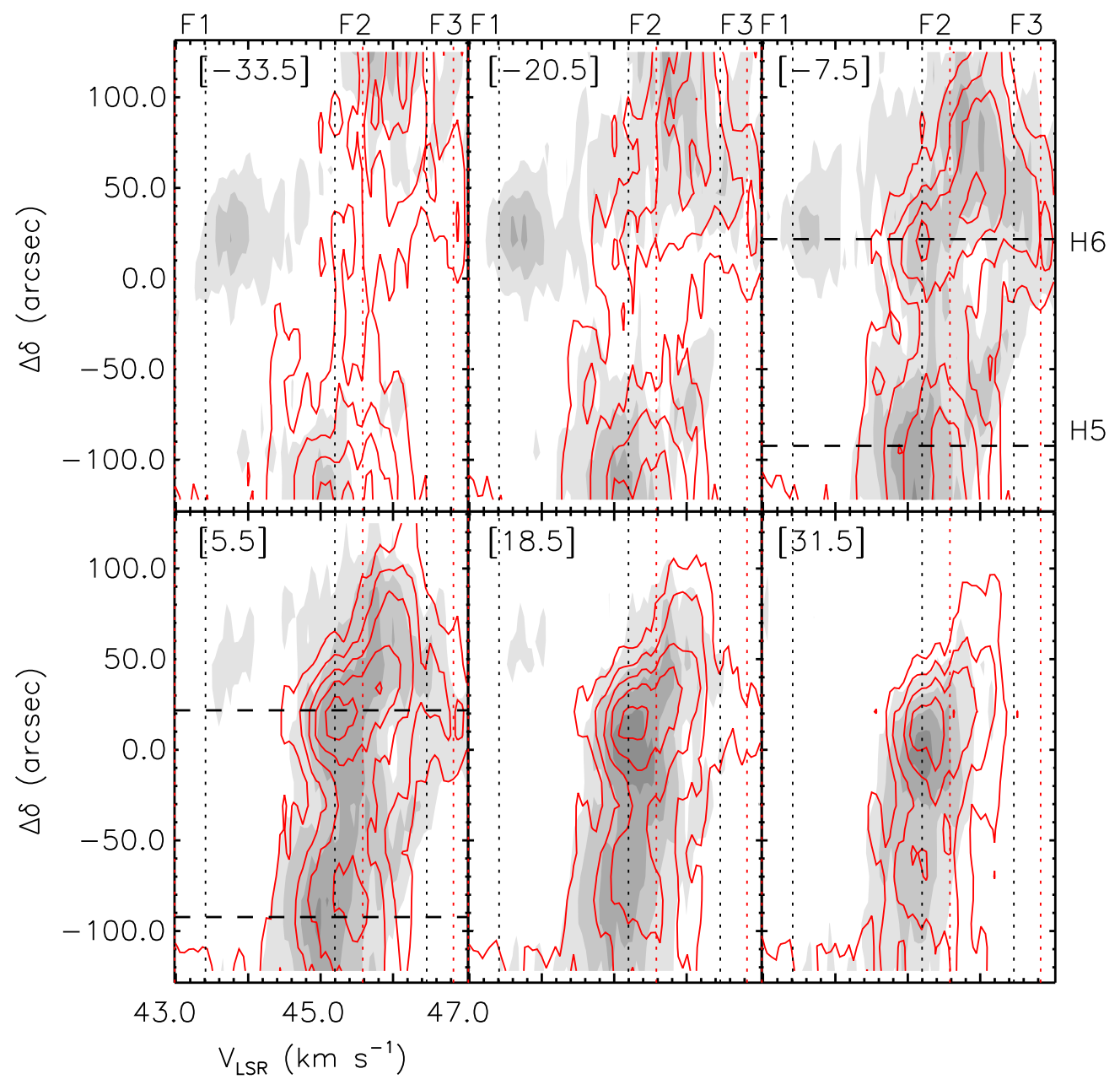

Figure 7. Position-Velocity diagrams of both (Red) $\mathrm{N}_{2} \mathrm{H}^{+}(1-0)\left(\mathrm{F}_{1}, \mathrm{~F}=0,1 \rightarrow 1,2\right)$ isolated component and (Grey-scale) $\mathrm{C}^{18} \mathrm{O}$ $(1-0)$. Cuts are taken from North to South through the cloud at each offset right ascension (which can be found in the top left of each panel). Emission in $\mathrm{N}_{2} \mathrm{H}^{+}(1-0)$ and $\mathrm{C}^{18} \mathrm{O}(1-0)$ is plotted from $3 \sigma\left(\sigma=0.14\right.$, and for $\mathrm{N}_{2} \mathrm{H}^{+}(1-0)$, $\left.\sigma=0.06\right)$. Contours increase in $3 \sigma$ steps. Vertical dotted lines refer to the centroid velocities derived from the GGF (see Appendix A). Horizontal dashed lines at offsets $\Delta \alpha=-7.5$ and 5.5 , refer to the positions of core H6 $(2,20)$, and core $\mathrm{H} 5(6,-86)$.

the component is seen to run from North-East to SouthEast, curving to the West toward the centre of the map. The $\mathrm{N}_{2} \mathrm{H}^{+}(1-0)$ emission in this component is restricted to the most southern portion of the cloud. The gas distribution changes shape between $44-46 \mathrm{~km} \mathrm{~s}^{-1}$ (Filament 2), peaking at the positions of the massive cores. Here, the $\mathrm{N}_{2} \mathrm{H}^{+}(1-0)$ follows the morphology of the $\mathrm{C}^{18} \mathrm{O}(1-0)$ more closely, and is extended over the whole filament, indicating the presence of widespread dense gas. Both species follow the shape of the extinction map, from North-West to the South, curving slightly to the East. Finally, another component is seen between $46-48 \mathrm{~km} \mathrm{~s}^{-1}$ (Filament 3). This emission is similar in morphology to the previous component. However, the emission is restricted to the northern portion of the cloud, and is not seen in the South. Given that this component has a well defined feature in both the spectra (Figure 5), and the channel maps (Figure 6), we consider this to be a separate filament.

Another way to inspect the gas kinematics across the cloud is through position-velocity (PV) diagrams. Figure7 shows the PV diagrams of both species $\left(\mathrm{N}_{2} \mathrm{H}^{+}(1-0)\left(\mathrm{F}_{1}, \mathrm{~F}\right.\right.$ $=0,1 \rightarrow 1,2)$ in red, and $\mathrm{C}^{18} \mathrm{O}(1-0)$ in grey scale), slicing the cloud from North to South, at each offset of right ascension in our $\mathrm{N}_{2} \mathrm{H}^{+}(1-0)$ map. Vertical dotted lines refer to the velocities of the individual components seen in both species as deduced by the Guided Gaussian Fit (GGF) of the average spectrum (see Appendix A for further details). At the most westerly point of the cloud $\left(\Delta \alpha=-33.5^{\prime \prime}\right)$, the $\mathrm{N}_{2} \mathrm{H}^{+}(1-0)$ emission follows the $\mathrm{C}^{18} \mathrm{O}(1-0)$ emission in both the North and South, except at $\Delta \delta \sim 30^{\prime \prime}$, where we can see an additional component in $\mathrm{C}^{18} \mathrm{O}(1-0)$ which is part of Filament 1. There is no significant velocity structure, except for a velocity change of about $1 \mathrm{~km} \mathrm{~s}^{-1}$ between the northern and southern emission on a scale of $\sim 1 \mathrm{pc}$, which corresponds to a very small velocity gradient of $\simeq$ $1 \mathrm{~km} \mathrm{~s}^{-1} \mathrm{pc}^{-1}$. Such a small gradient is comparable to those found in low-mass cores (e.g. Caselli et al. 2002a), but here it is seen over a significantly larger extent. 

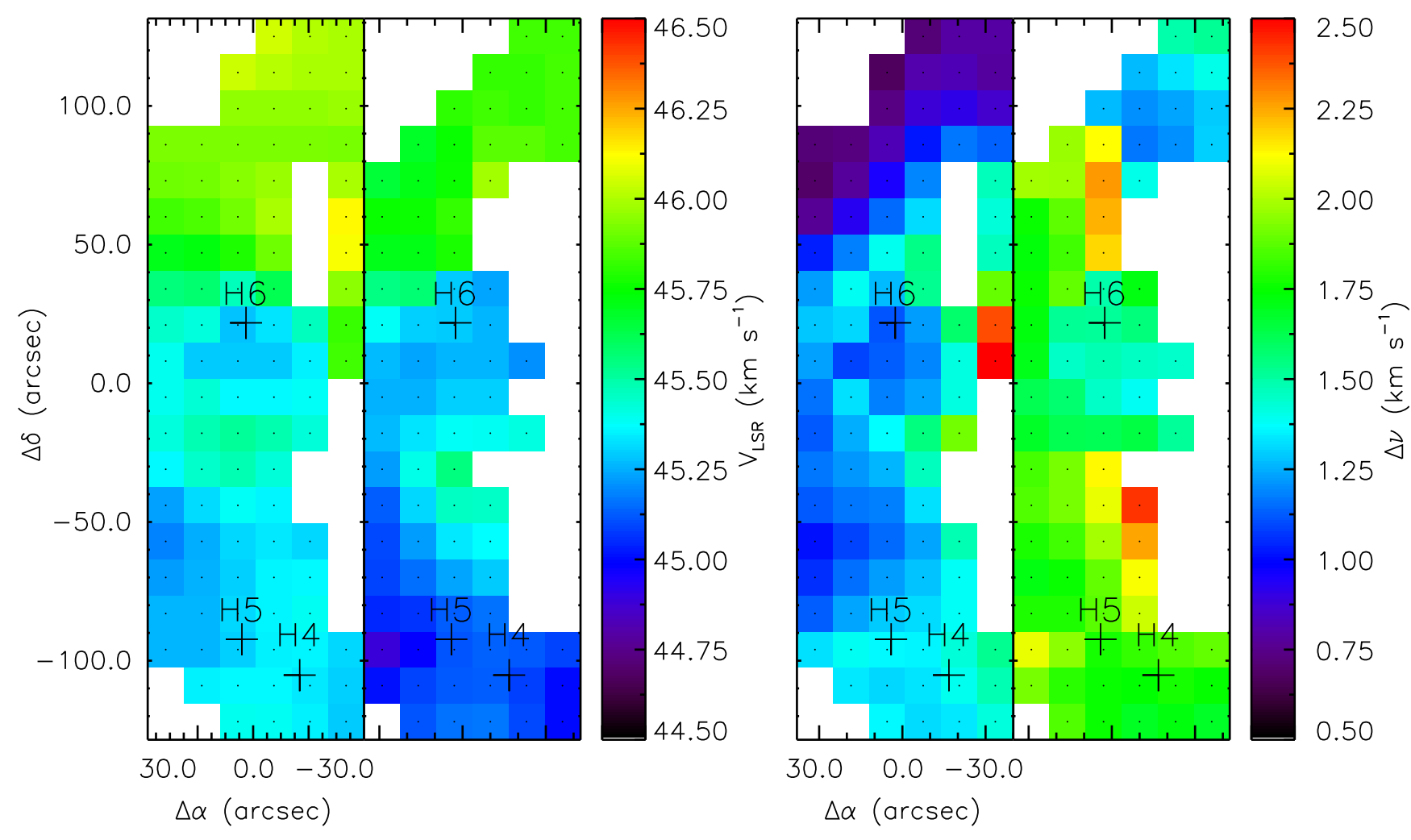

Figure 8. $\mathrm{V}_{L S R}$ and $\Delta v$ maps of Filament 2, as derived from the Guided Gaussian Fits (see Appendix A). Maps on the left represent the $\mathrm{V}_{L S R}$ of (Left) $\mathrm{N}_{2} \mathrm{H}^{+}(1-0)$ and (Right) $\mathrm{C}^{18} \mathrm{O}(1-0)$. Maps on the right represent the linewidth, $\Delta v$ of $($ Left $) \mathrm{N}_{2} \mathrm{H}^{+}(1-0)$ and (Right) $\mathrm{C}^{18} \mathrm{O}(1-0)$. Black crosses indicate the positions of the massive cores from BT12.

As we move towards the centre of the cloud, the emission becomes stronger ( $\max$ intensity of $\mathrm{N}_{2} \mathrm{H}^{+}(1-0)\left(\mathrm{F}_{1}\right.$, $\mathrm{F}=0,1 \rightarrow 1,2)$ at $-33.5^{\prime \prime}=0.82 \mathrm{~K} \mathrm{~km} \mathrm{~s}^{-1}$ compared to $1.25 \mathrm{~K} \mathrm{~km} \mathrm{~s}^{-1}$ at $5.5^{\prime \prime}$ ) and spreads over a larger velocity range. This can be seen towards offsets $\Delta \alpha=-20.5^{\prime \prime}$ and $-7.5^{\prime \prime}$, where an additional component at higher velocities (Filament 3) appears (most prominent between $0^{\prime \prime}<\Delta \delta$ $\left.<50^{\prime \prime}\right)$. Core H6 lies between $-7.5^{\prime \prime}<\Delta \alpha<5.5^{\prime \prime}$ at $\Delta \delta=$ $20^{\prime \prime}$. Here all three filaments are seen, with Filament 2 being the most prominent in $\mathrm{N}_{2} \mathrm{H}^{+}(1-0)$. The channel maps of Figure 6 also show that these features coincide spatially at the position of core H6, suggestive of filament merging (see below).

\subsubsection{Centroid velocity and line width}

Figure 8 shows the centroid velocity map of Filament 2 in $\mathrm{N}_{2} \mathrm{H}^{+}(1-0)\left(\mathrm{F}_{1}, \mathrm{~F}=0,1 \rightarrow 1,2\right)$ and $\left.\mathrm{C}^{18} \mathrm{O}(1-0)\right)^{2}$. These velocities are derived from the GGF (Guided Gaussian Fit) method (Appendix A). The velocity field appears coherent, except for a velocity change of $\sim 0.5 \mathrm{~km} \mathrm{~s}^{-1}$ at the

2 Both the low and high velocity components (Filament 1 and 3, respectively) do not cover a large enough area in $\mathrm{N}_{2} \mathrm{H}^{+}(1-0)$ to see any significant velocity gradient, so we do not consider the other two filaments in this analysis. northern edge of core H6. The change in velocity from $\sim$ $45.9 \mathrm{~km} \mathrm{~s}^{-1}$ (North) to $\sim 45.4 \mathrm{~km} \mathrm{~s}^{-1}$ (South) occurs within $\sim$ one beam width, corresponding to a local velocity gradient of $\simeq 1 \mathrm{~km} \mathrm{~s}^{-1} \mathrm{pc}^{-1}$. Same conclusions are drawn from the $\mathrm{C}^{18} \mathrm{O}(1-0)$ line, although the velocity field appears more complex south of core H6. Thus, the abrupt (but relatively small) velocity change is present in the dense material as well as in the lower density envelope, suggesting that it originates from the larger scale.

Figure 8 also shows the $\mathrm{N}_{2} \mathrm{H}^{+}(1-0)\left(\mathrm{F}_{1}, \mathrm{~F}=0,1 \rightarrow\right.$ $1,2)$ and $\mathrm{C}^{18} \mathrm{O}(1-0)$ line width maps of Filament 2 . The first thing to note is that line widths are dominated by nonthermal motions. In fact, adopting an average kinetic temperature of $15 \mathrm{~K}$ (e.g. Ragan et al. 2011), the thermal width for both species is $0.15 \mathrm{~km} \mathrm{~s}^{-1}$. Thus, the observed widths are between 3 and 15 times the thermal width, with the narrowest widths found toward the North. On average, $\mathrm{C}^{18} \mathrm{O}$ $(1-0)$ and $\mathrm{N}_{2} \mathrm{H}^{+}(1-0)$ line widths have similar values. This is in striking contrast to low-mass star-forming regions, where lines of $\mathrm{C}^{18} \mathrm{O}(1-0)$ typically show broader line widths compared to those measured in high density tracers (by a factor between 1.5 and 2; e.g. Fuller \& Muers 1992). However, this is consistent with our finding that $\mathrm{N}_{2} \mathrm{H}^{+}$is widespread across the filament and not preferentially tracing dense cores as in low-mass star-forming regions (see Figure 2). In both species, the line widths get narrower towards the northern 


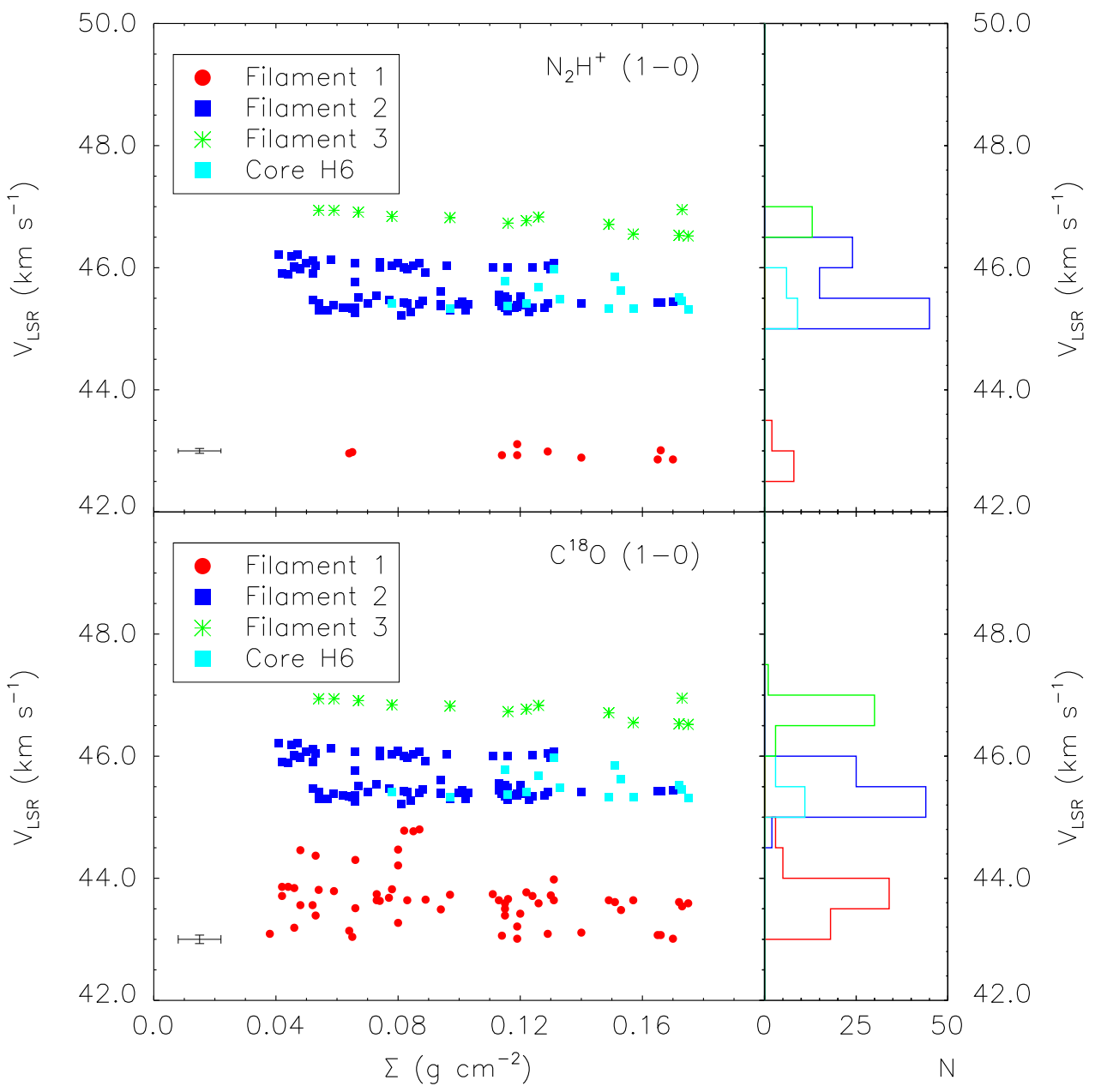

Figure 9. $V_{\text {LSR }}$ as a function of mass surface density for (Top) $\mathrm{N}_{2} \mathrm{H}^{+}(1-0)$ and (Bottom) $\mathrm{C}^{18} \mathrm{O}(1-0)$. The right of each plot shows a histogram of the $\mathrm{V}_{\mathrm{LSR}}$ information. The uncertainty in the $\mathrm{V}_{\mathrm{LSR}}$ are derived from the Gaussian fits and their mean values overall the three filaments are $\simeq 0.07 \mathrm{~km} \mathrm{~s}^{-1}$ and $0.04 \mathrm{~km} \mathrm{~s}^{-1}$ for $\mathrm{C}^{18} \mathrm{O}$ and $\mathrm{N}_{2} \mathrm{H}^{+}$, respectively. Errors in the mass surface density represent the $1 \sigma$ level $=0.007 \mathrm{~g} \mathrm{~cm}^{-2}$. Mean uncertainties are dipicted in the bottom left-hand corner of each plot.

portion of the cloud and (to a lesser extent) toward the starless core H6, indicating the presence of relatively quiescent (and probably pristine) gas.

Figure 9 displays the $\mathrm{V}_{L S R}$ of $\mathrm{N}_{2} \mathrm{H}^{+}(1-0)\left(\mathrm{F}_{1}, \mathrm{~F}=\right.$ $0,1 \rightarrow 1,2)$ and $\mathrm{C}^{18} \mathrm{O}(1-0)$ as a function of mass surface density. The three filaments are clearly seen as well separated velocity components (as determined by the Gaussian fits). The average velocities of the individual components differ slightly between the two species. In Filament 1, we find a mean $\mathrm{V}_{L S R}$ of $42.95(0.17) \mathrm{km} \mathrm{s}^{-1}$ in $\mathrm{N}_{2} \mathrm{H}^{+}(1-0)$, and $43.65(0.12) \mathrm{km} \mathrm{s}^{-1}$ in $\mathrm{C}^{18} \mathrm{O}(1-0)$. Filament 2 has a mean velocity of $45.63(0.03) \mathrm{km} \mathrm{s}^{-1}$ in $\mathrm{N}_{2} \mathrm{H}^{+}(1-0)$, and $45.40(0.03) \mathrm{km} \mathrm{s}^{-1}$ in $\mathrm{C}^{18} \mathrm{O}(1-0)$. Filament 3 has a mean velocity of $46.77(0.06) \mathrm{kms}^{-1}$ in $\mathrm{N}_{2} \mathrm{H}^{+}(1-0)$, and 46.76 $(0.05) \mathrm{km} \mathrm{s}^{-1}$ in $\mathrm{C}^{18} \mathrm{O}(1-0)$. We note that at mass surface densites below $0.06 \mathrm{~g} \mathrm{~cm}^{-2}$, the $\mathrm{C}^{18} \mathrm{O}$ filaments span a broader range of velocities. Moreover, the relative velocity between Filament 2 and 1 is larger than that between Filament 2 and 3. This could be at the origin of the observed velocity shift between $\mathrm{N}_{2} \mathrm{H}^{+}$and $\mathrm{C}^{18} \mathrm{O}$ in Filament 2 (see Figure[5 and sections 3.2 .3 and 4 for more details). The abrupt change in velocity observed in Filament 2 (Figure 7 and (8) is seen here as the "gap" between the two groups of blue points. It is interesting to see that the velocities associated with the massive starless core $\mathrm{H} 6$ are filling this gap, suggesting that this core has probably formed at the interface of material moving at different velocities.

\subsubsection{The $\mathrm{N}_{2} \mathrm{H}^{+}-\mathrm{C}^{18} \mathrm{O}$ velocity shift}

Figures 10 and 11 make clear that the velocity shift seen between $\mathrm{N}_{2} \mathrm{H}^{+}(1-0)$ and $\mathrm{C}^{18} \mathrm{O}(1-0)$ is the result of largescale kinematics. Figure 10 is a map of the $\mathrm{N}_{2} \mathrm{H}^{+}-\mathrm{C}^{18} \mathrm{O}$ velocity shift measured across Filament 2, with the contours of $\mathrm{SiO}(2-1)$ overlapped on top. The $\mathrm{N}_{2} \mathrm{H}^{+}(1-0)$ emission is largely red-shifted with respect to the $\mathrm{C}^{18} \mathrm{O}(1-0)$ emission, and we report a mean velocity shift of $0.18 \pm 0.04 \mathrm{~km} \mathrm{~s}^{-1}$, across the whole filament. The largest velocity shifts are seen to the North and South of the cloud. North of offset $\Delta \delta=$ 86.0 , the average velocity shift is $0.22(0.04) \mathrm{km} \mathrm{s}^{-1}$. South 


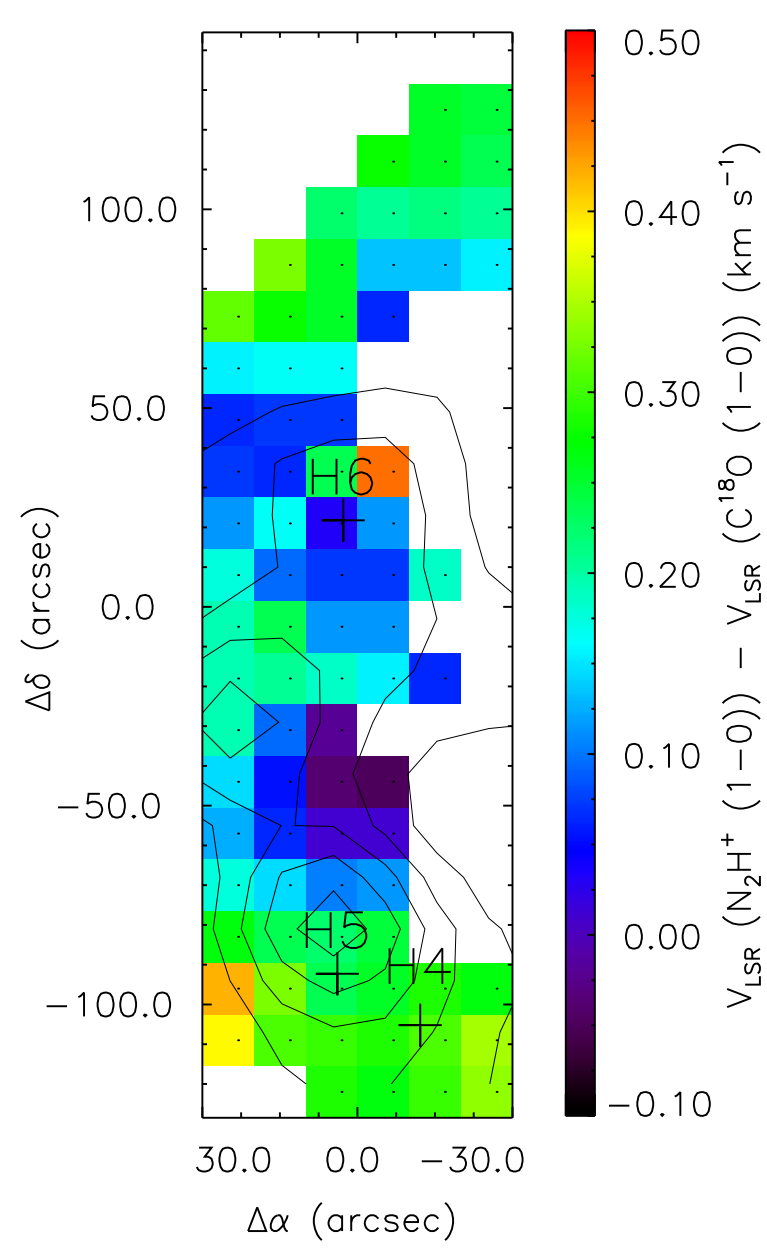

Figure 10. Map of the velocity shift between $\mathrm{N}_{2} \mathrm{H}^{+}(1-0)$ and $\mathrm{C}^{18} \mathrm{O}(1-0)$ shown for Filament 2 only. Overlaid in black contours is the $\mathrm{SiO}(2-1)$ emission. Contours are $3 \sigma$ to $0.5 \mathrm{~K} \mathrm{~km} \mathrm{~s}^{-1}$ in steps of $3 \sigma\left(\sim 0.1 \mathrm{~K} \mathrm{~km} \mathrm{~s}^{-1}\right)$. Black crosses refer to the positions of the massive cores from BT12.

of offset $\Delta \delta=-70.0^{\prime \prime}$, the velocity shift is $0.26(0.04) \mathrm{km} \mathrm{s}^{-1}$. Between these offsets, the velocity shift is $0.13(0.04) \mathrm{km} \mathrm{s}^{-1}$. No correlation is found between the velocity shift and the $\mathrm{SiO}(2-1)$ integrated intensity. The velocity shift is also clear in Figure 11, which shows the velocity difference between $\mathrm{C}^{18} \mathrm{O}$ and $\mathrm{N}_{2} \mathrm{H}^{+}$as a function of mass surface density. Also in this case, no correlation is found, but the shift is clearly present all across the filament.

The velocity shift is not constant along the filament. Figure 12 shows the $\mathrm{N}_{2} \mathrm{H}^{+}-\mathrm{C}^{18} \mathrm{O}$ velocity difference as a function of declination along the four central strips at fixed right ascension offsets $-7.5^{\prime \prime}, 5.5^{\prime \prime}, 18.5^{\prime \prime}$ and $31.5^{\prime \prime}$. From this figure it is clear that the shifts in the North and South have similar magnitudes, while variations are present in between. The variations along the four strips appear similar up to the H6 core, where the outer strip at $\Delta \alpha=-7.5^{\prime \prime}$ (and to a lesser extent the strip passing through the peak of core H6) show different behavior. This is the region where the abrupt change in velocity has been found (see Figure8). Those regions where the dense gas and its envelope appear to be moving with the same velocity are present at $\Delta \delta=$

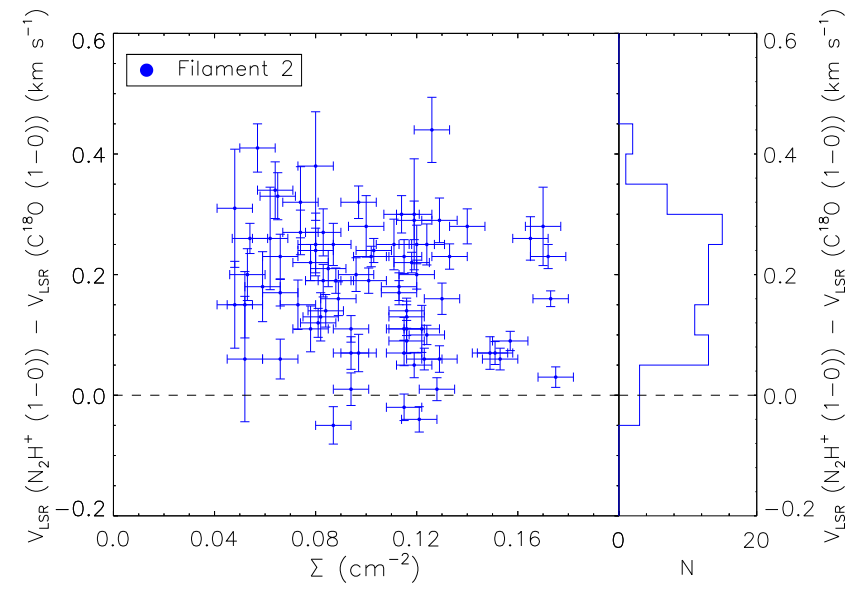

Figure 11. Velocity shift between $\mathrm{N}_{2} \mathrm{H}^{+}(1-0)$ and $\mathrm{C}^{18} \mathrm{O}(1-0)$ as a function of mass surface density. The right of the plot shows a histogram of this velocity shift information. Errors in the mass surface density represent an assumed uncertainty of $0.007 \mathrm{~g} \mathrm{~cm}^{-2}$.

$-40^{\prime \prime}$ in the two western strips and at the peak of core H6. However these points of minimum shift are surrounded by sharp variations, especially evident toward core H6. Discussion on a possible origin of this velocity shift can be found in section 4

\subsection{The $\mathrm{C}^{18} \mathrm{O} / \mathrm{N}_{2} \mathrm{H}^{+}$integrated intensity ratio}

Tafalla \& Santiago (2004) used CO depletion as a chemical clock, to indicate an evolutionary sequence of individual cores. This is achieved by taking two species that show different amounts of depletion at high densities. $\mathrm{C}^{18} \mathrm{O}$ freezeout becomes heavy at densities upwards of a few $10^{4} \mathrm{~cm}^{-3}$, whereas $\mathrm{N}_{2} \mathrm{H}^{+}(1-0)$ remains in the gas phase up to densities $\geqslant 10^{5} \mathrm{~cm}^{-3}$ (e.g., Caselli et al. 1999, Tafalla et al. 2002). Moreover, $\mathrm{N}_{2} \mathrm{H}^{+}$takes longer than $\mathrm{C}^{18} \mathrm{O}$ to form, due to the slower neutral-neutral reactions involved in the production of $\mathrm{N}_{2}$, the parent species of $\mathrm{N}_{2} \mathrm{H}^{+}$(e.g. Hily-Blant et al. 2010). Therefore, an early stage of evolution would be characterised as having an undepleted $\mathrm{C}^{18} \mathrm{O}$ and low abundances of $\mathrm{N}_{2} \mathrm{H}^{+}$. As the cores follow their evolution toward star formation, their density increases and $\mathrm{C}^{18} \mathrm{O}$ starts to freeze-out. Thus, with time, the $\mathrm{CO}$ abundance falls while $\mathrm{N}_{2} \mathrm{H}^{+}$abundances reach equilibrium and maintain relatively large values up to volume densities of $\geqslant 10^{5} \mathrm{~cm}^{-3}$, as the freeze-out of $\mathrm{CO}$ initially boosts the production rate of $\mathrm{N}_{2} \mathrm{H}^{+}$(given that $\mathrm{CO}$ is an important destruction partner of $\mathrm{N}_{2} \mathrm{H}^{+}$). In light of this, we can use the method of Tafalla \& Santiago (2004) and take the ratio of the integrated intensities of $\mathrm{C}^{18} \mathrm{O}(1-0)$ and $\mathrm{N}_{2} \mathrm{H}^{+}(1-0)$ :

$$
R \equiv\left[\frac{I\left(\mathrm{C}^{18} \mathrm{O}\right)}{I\left(\mathrm{~N}_{2} \mathrm{H}^{+}\right)}\right],
$$

to define a chemical clock. Chemically young gas is characterized by values of $R>1$. Figure 13 displays the map of the $\mathrm{C}^{18} \mathrm{O} / \mathrm{N}_{2} \mathrm{H}^{+}$intensity ratio integrated between 44 and $46 \mathrm{~km} \mathrm{~s}^{-1}$, where Filament 2 is more prominent. To obtain this map, we first smoothed the $\mathrm{C}^{18} \mathrm{O}(1-0)$ to $26^{\prime \prime}$, the angular resolution of the $\mathrm{N}_{2} \mathrm{H}^{+}(1-0)$ map, and regridded 
the $\mathrm{C}^{18} \mathrm{O}(1-0)$ map. The $\mathrm{C}^{18} \mathrm{O} / \mathrm{N}_{2} \mathrm{H}^{+}$integrated intensity ratio has a value $<1$ throughout the whole filament, except for the northern regions. The bulk of the filament shows large volume of $\mathrm{N}_{2} \mathrm{H}^{+}$with respect to $\mathrm{C}^{18} \mathrm{O}$, and this suggests that the gas is in a chemically evolved state. This is clearly seen in Figure 14, where $R$ is plotted versus the mass surface density: no $R$ values larger than 1.0 are found at $\Sigma \geqslant 0.09 \mathrm{~g} \mathrm{~cm}^{-2}$. Given that the chemical time scale is inversely dependent on the number density, the observed advanced chemical evolution is probably just the result of the relatively large average density of the filament when compared to low-mass star-forming regions (see Section 3.5). Only in the North, the gas appears relatively less chemically evolved, suggesting the presence of lower density material.

\subsection{Column Density}

The $\mathrm{N}_{2} \mathrm{H}^{+}$and $\mathrm{C}^{18} \mathrm{O}$ column densities have been calculated from the integrated intensity of the $\mathrm{N}_{2} \mathrm{H}^{+}(1-0) \mathrm{F}_{1}, \mathrm{~F}=$ $0,1 \rightarrow 1,2$ hyperfine component (divided by its statistical weight) and $\mathrm{C}^{18} \mathrm{O}(1-0)$ emission. The assumption here is that both lines are optically thin. This has been verified for the $\mathrm{N}_{2} \mathrm{H}^{+}$isolated component (with statistical weight of $\simeq 0.11111$; Caselli et al. 1995) with the HFS fitting technique (see Appendix B) and for $\mathrm{C}^{18} \mathrm{O}(1-0)$, Paper II). To measure the column densities, we then used equation (A4) in Caselli et al. (2002).

The average $\mathrm{N}_{2} \mathrm{H}^{+}$column density across the IRDC is $\mathrm{N}\left(\mathrm{N}_{2} \mathrm{H}^{+}\right)=(1.14 \pm 0.11) \times 10^{13} \mathrm{~cm}^{-2}$, peaking in the South at offset $\left(5.5^{\prime \prime},-122^{\prime \prime}\right)$, with a value of $(2.33 \pm 0.25)$ $\times 10^{13} \mathrm{~cm}^{-2}$. The minimum value of $\mathrm{N}\left(\mathrm{N}_{2} \mathrm{H}^{+}\right)$is located in the North at offset $\left(5.5^{\prime \prime}, 125^{\prime \prime}\right)$, where $\mathrm{N}\left(\mathrm{N}_{2} \mathrm{H}^{+}\right)=$ $(2.33 \pm 0.69) \times 10^{12} \mathrm{~cm}^{-2}$. These column densities are similar to those measured toward low-mass cores (Caselli et al. 2002a), indicating that the average physical and chemical conditions of the whole filament traced by $\mathrm{N}_{2} \mathrm{H}^{+}(1-0)$ are similar to those of the well-known dense cores in nearby lowmass star-forming regions, in agreement with the study of Vasyunina et al. (2011).

The average $\mathrm{C}^{18} \mathrm{O}$ column density throughout the filament is $\mathrm{N}\left(\mathrm{C}^{18} \mathrm{O}\right)=(3.82 \pm 0.04) \times 10^{15} \mathrm{~cm}^{-2}$, peaking at position $(-10.0,34.0)$, North-West of the extinction peak of core H6 with a value of $(5.95 \pm 0.72) \times 10^{15} \mathrm{~cm}^{-2}$. This is consistent with Paper II, that found evidence of CO freezeout toward the center of H6.

We find a significant correlation $(\mathrm{r}$-value $=0.8)$ between the column density of $\mathrm{N}_{2} \mathrm{H}^{+}(1-0)$ and the mass surface density (as expected given the strong correlation found in Figure 4 between the $\mathrm{N}_{2} \mathrm{H}^{+}(1-0)$ integrated intensity and the mass surface density $): \mathrm{N}\left(\mathrm{N}_{2} \mathrm{H}^{+}\right)=(1.5 \pm 0.2) \times 10^{12}$ $+(9.8 \pm 0.3) \times 10^{13} \Sigma$. This correlation implies a constant fractional abundance of $\mathrm{N}_{2} \mathrm{H}^{+}$(w.r.t. $\mathrm{H}_{2}$ molecules) of $\simeq 4 \times 10^{-10}$, similar to the value found in low-mass cores (a few times $10^{-10}$; Caselli et al. 2002b, Crapsi et al. 2005) and in the quiescent massive starless core in Orion (Orion B9; Miettinen et al. 2012), but lower than the value measured in the direction of massive IR-dark clumps (a few $\times 10^{-9}$; Miettinen et al. 2011). Quiescent dense core conditions once again appear to be appropriate in describing G035.39-00.33.

\subsection{Number density and kinetic temperature}

Observations of the $J=1 \rightarrow 0$ and $J=3 \rightarrow 2$ rotational transitions of $\mathrm{N}_{2} \mathrm{H}^{+}$can constrain the number density, if the gas temperature is known (see Section 3.1). No gas temperature measurements have been made yet across G035.3900.33 , so we assume a constant temperature of $15 \mathrm{~K}$ across the cloud, based on the kinetic temperature derived in other IRDCs (e.g. Pillai et al. 2006, Ragan et al. 2011), and close to the dust temperature measured across the filament by Nguyen Luong et al. (2011) using Herschel data.

To calculate the number density, the non-LTE radiative transfer code RADEX (van der Tak et al. 2007) was used. The $\mathrm{N}_{2} \mathrm{H}^{+}(3-2)$ map was smoothed to $26^{\prime \prime}$ resolution, to match that of the $\mathrm{N}_{2} \mathrm{H}^{+}(1-0)$ data. Given the complexity of the spectra and the fact that the $(3-2)$ transition does not have isolated hyperfine components, we fitted a single Gaussian peak to the isolated hyperfine component of the $\mathrm{N}_{2} \mathrm{H}^{+}(1-0)$ irrespective of the fact that we may see multiple components along the line of sight. In addition to this, we performed a HFS fit assuming optically thin conditions to the $\mathrm{N}_{2} \mathrm{H}^{+}(3-2)$ lines in CLASS, which gives, as one of its outputs, the linewidth. The average linewidth between the $\mathrm{N}_{2} \mathrm{H}^{+}(1-0)$ and $(3-2)$ profiles was chosen as input parameter for the RADEX calculation. The column density input to RADEX was calculated using the integrated intensity of $\mathrm{N}_{2} \mathrm{H}^{+}(1-0)$ over the velocity range $40-50 \mathrm{~km} \mathrm{~s}^{-1}$ (first using the isolated component and then scaling by the statistical weight). As a final assumption we then used a value of $15 \mathrm{~K}$ for the kinetic temperature of the gas. The number density was then varied between $1 \times 10^{3} \mathrm{~cm}^{-3}$ and $1 \times 10^{8} \mathrm{~cm}^{-3}$ and a minimisation technique was used to find the best fit to the observed brightness temperature ratio of the $J=3 \rightarrow 2$ and $J=1 \rightarrow 0$ lines. Figure 15 shows the result of this minimization technique, i.e. the number density map of G035.39-00.33. The average $\mathrm{H}_{2}$ number density across the filament (measured with a $26^{\prime \prime}$ beam, corresponding to a linear size of $0.36 \mathrm{pc}$ ) is $\simeq 5 \times 10^{4} \mathrm{~cm}^{-3}$, with peaks close to the three massive cores and East of core H6. This value is roughly a factor of 10 larger than that found using extinction mapping in Paper III. Possible causes of this difference are unresolved clumping and the fact that the analysis done with the $\mathrm{N}_{2} \mathrm{H}^{+}$data only includes those points where the 3-2 line is observed, therefore it is biased toward higher densities (as the 3-2 line has a critical density of $\left.3 \times 10^{6} \mathrm{~cm}^{-3}\right)$.

To check possible temperature variations across the filament, we fix the number density to the average value found with the previous method and find the kinetic temperature map. Figure[16 shows the kinetic temperature map obtained from the $\mathrm{N}_{2} \mathrm{H}^{+}(3-2) /(1-0)$ intensity ratio and the RADEX code, assuming a constant number density across the filament of $5 \times 10^{4} \mathrm{~cm}^{-3}$ (the average value found in Figure 15). A temperature gradient is present perpendicular to the filament, with the largest values $(\sim 40 \mathrm{~K})$, close to the central $\mathrm{SiO}$ peak (see Figure 10). Although this analysis should be taken with caution, given the simplistic assumption of constant density, we speculate that the observed temperature increase may be linked to the filament merging and the consequent shock which has produced the extended $\mathrm{SiO}$ emission (Paper I). In fact, the extinction in these regions is still large enough to exclude any temperature enhancement due 


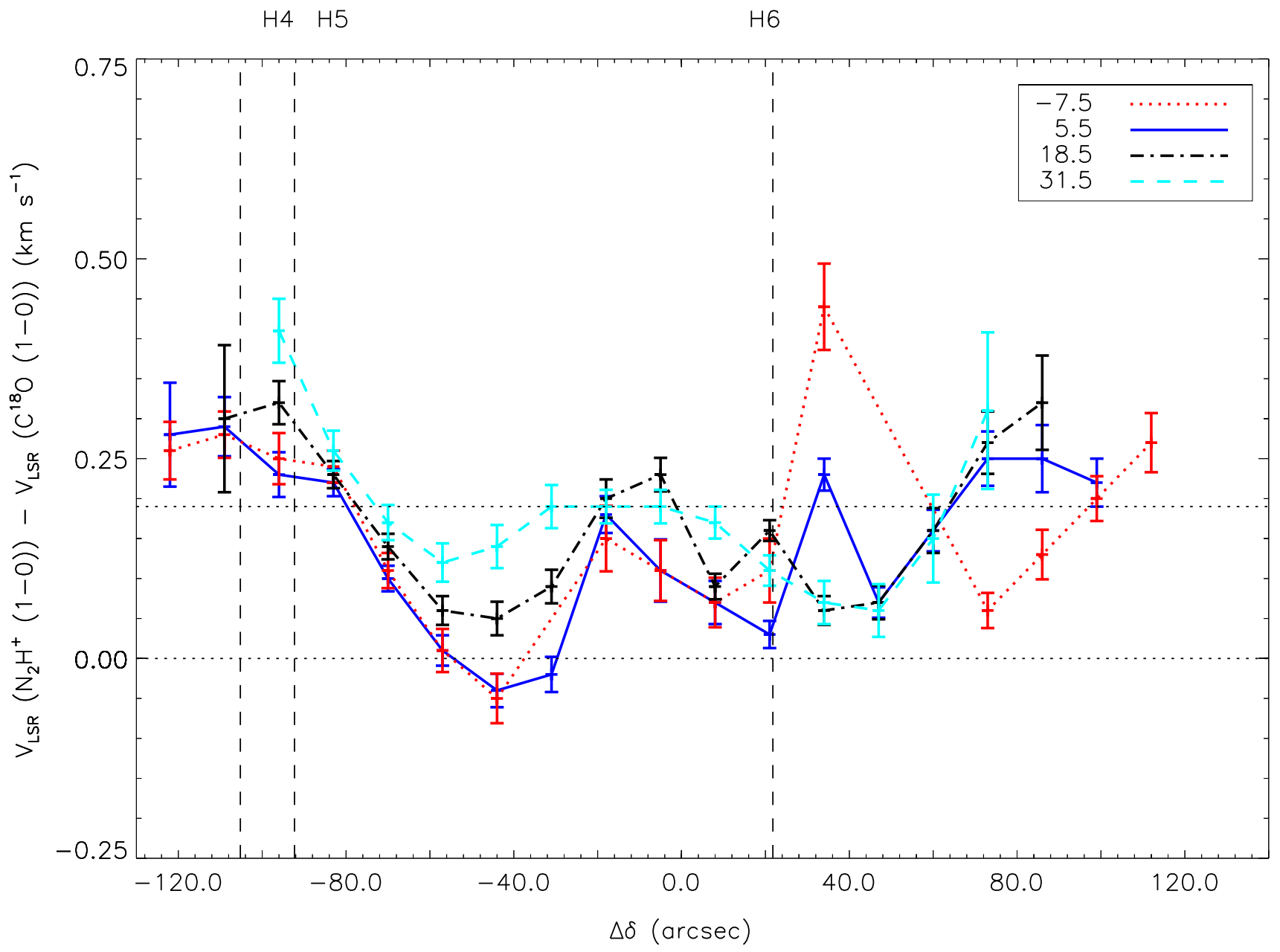

Figure 12. Velocity shift between $\mathrm{N}_{2} \mathrm{H}^{+}(1-0)$ and $\mathrm{C}^{18} \mathrm{O}(1-0)$ as a function of offset in declination along four strips of constant right ascension (see legend in top right). The blue line refers to the strip passing closer to the center of core H6. Horizontal dotted lines correspond to the mean values of the velocity shift, $0.18 \pm 0.04 \mathrm{~km} \mathrm{~s}^{-1}$, and the zero line. Vertical dotted lines indicate the positions in offset declination of the three massive cores from BT12.

to the interstellar radiation field. Independent temperature measurements (e.g. from the inversion transitions of ammonia) and higher angular resolution observations are needed to put more stringent constraints on the temperature and density structure across G035.39-00.33.

\section{DISCUSSION}

\subsection{What is causing the $\mathrm{N}_{2} \mathrm{H}^{+}-\mathrm{C}^{18} \mathrm{O}$ velocity shift?}

The velocity shift between the $\mathrm{N}_{2} \mathrm{H}^{+}(1-0)$, and $\mathrm{C}^{18} \mathrm{O}(1-0)$ reveals relative motions between the dense gas (traced by $\mathrm{N}_{2} \mathrm{H}^{+}$) and the surrounding less dense envelope (traced by $\mathrm{C}^{18} \mathrm{O}$ ). This shift has been searched for but not found in lowmass star-forming regions (e.g. Walsh et al. 2004, Kirk et al. 2007, Hacar \& Tafalla 2011). Hacar \& Tafalla (2011) studied the centroid velocities of $\mathrm{N}_{2} \mathrm{H}^{+}(1-0)$ and $\mathrm{C}^{18} \mathrm{O}(1-0)$ in $\mathrm{L} 1517$, and found a good match between the two tracers. They use this to say that there are no significant motions between the different density regimes of the cloud, namely the dense cores as traced by $\mathrm{N}_{2} \mathrm{H}^{+}(1-0)$ and the surrounding envelope traced by $\mathrm{C}^{18} \mathrm{O}(1-0)$. They go on to conclude that this implies that the velocity structure of the core is therefore not an intrinsic property of the core, but a result of large-scale motions on filamentary scales. In the case of G035.39-00.33, $\mathrm{N}_{2} \mathrm{H}^{+}(1-0)$ traces the dense cores as well as the filament over a distance of $\sim 4 \mathrm{pc}$, similar to the $\mathrm{C}^{18} \mathrm{O}$ $(1-0)$ line, so that the observed velocity shift represents a puzzle which needs to be investigated in more detail.

The channel maps in Figure6 clearly show that significant $\mathrm{N}_{2} \mathrm{H}^{+}(1-0)$ emission is present in a narrower range of velocities compared to the $\mathrm{C}^{18} \mathrm{O}(1-0)$ emission. In particular, at the lowest velocities identified with Filament 1 (between 42 and $44 \mathrm{kms}^{-1}$ ), $\mathrm{N}_{2} \mathrm{H}^{+}$is practically undetected along most of the filament, whereas Filament 3 (between about 46 and $48 \mathrm{~km} \mathrm{~s}^{-1}$ ) is also seen in $\mathrm{N}_{2} \mathrm{H}^{+}(1-0)$ up to $47 \mathrm{~km} \mathrm{~s}^{-1}$. If the three identified filaments are interacting, the velocity field within the denser Filament 2 will then be affected by Filament 1 and 3 in the case of $\mathrm{C}^{18} \mathrm{O}(1-0)$, but only by Filament 3 in the case of $\mathrm{N}_{2} \mathrm{H}^{+}(1-0)$. This would imply a blueshift of the $\mathrm{C}^{18} \mathrm{O}$ line, as we indeed observe 


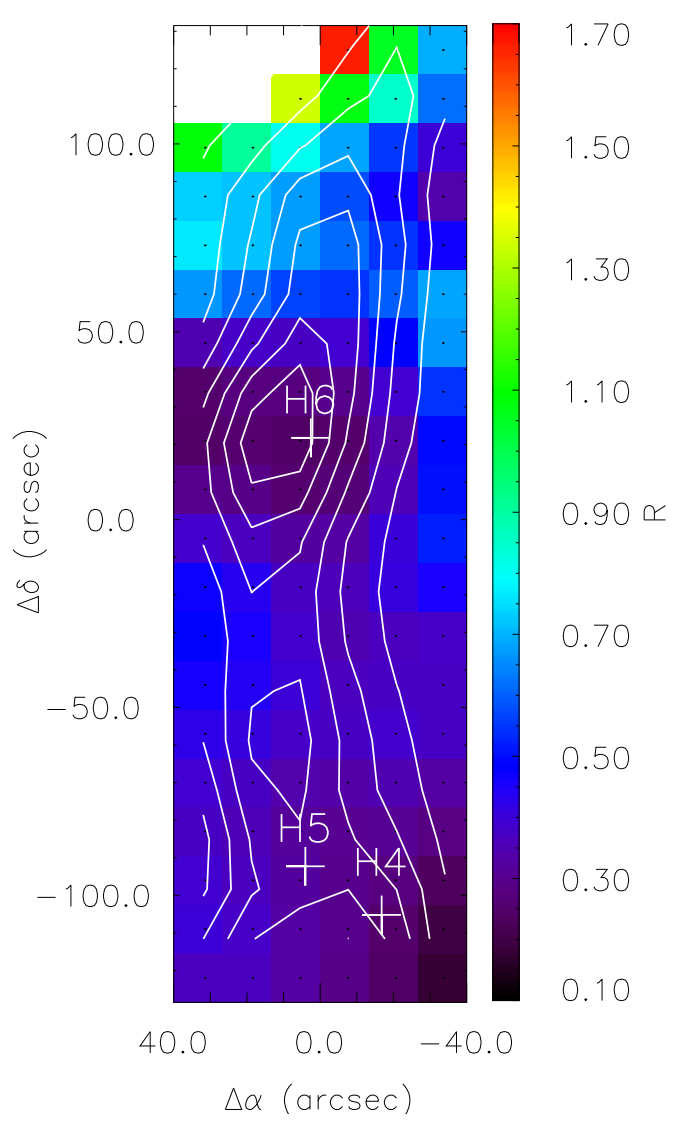

Figure 13. Map of the integrated intensity ratio, $\mathrm{R} \equiv$ $\left[I\left(\mathrm{C}^{18} \mathrm{O}\right) / I\left(\mathrm{~N}_{2} \mathrm{H}^{+}\right)\right]$. Intensities integrated between 44 and 46 $\mathrm{km} \mathrm{s}^{-1}$. Here, the integrated intensity of the isolated component of $\mathrm{N}_{2} \mathrm{H}^{+}(1-0)$ is scaled up by its statistical weight, such that it is representative of the total integrated intensity. Overlaid on top are contours of the mass surface density smoothed to a $26^{\prime \prime}$ beam. Contour levels are $3 \sigma\left(\sigma \sim 0.021 \mathrm{gcm}^{-2}\right)$, increasing in steps of $3 \sigma$. Crosses indicate the positions of the three massive cores are overlaid from BT12.

(see Figure 11). The filament interaction is also evident in Figure 9, where the $\mathrm{C}^{18} \mathrm{O}$ panel shows less distinct velocity components, and Filament 2 is split in two velocity components parallel to Filaments 1 and 3, with the densest region in the IRDC (core H6) in between. The abrupt change in velocity seen at the edge of core H6 (Figure 8) could also be produced by filament merging, with the consequent formation of a dense core at the intersection. Finally, Figure12 displays a variation of the observed velocity shift between the $\mathrm{C}^{18} \mathrm{O}(1-0)$ and $\mathrm{N}_{2} \mathrm{H}^{+}(1-0)$ lines along Filament 2, which can be explained by inhomogeneities along the interacting Filaments 1 and 3. Indeed, looking at Figures 6] 10 and 12 one can see that the maxima in the velocity shift seen in Filament 2 correspond to local maxima of the $\mathrm{C}^{18} \mathrm{O}(1-0)$ intensity in Filament 1. At these positions the densities are expected to be higher (further indicated by the presence of $\left.\mathrm{N}_{2} \mathrm{H}^{+}(1-0)\right)$ and thus the interaction more prominent.

The above observational evidence suggests that Filament 2 may be the result of the merging of Filaments 1 and 3, as sketched in Figure 17 This process of filament merging is still ongoing, with material from Filament 1 still mov-

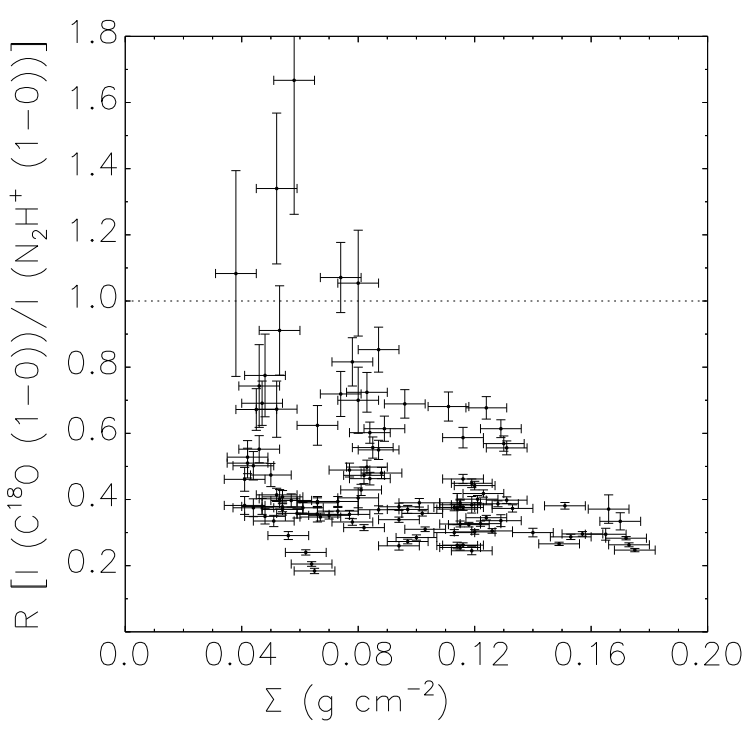

Figure 14. Integrated intensity ratio, $\mathrm{R} \equiv\left[I\left(\mathrm{C}^{18} \mathrm{O}\right) / I\left(\mathrm{~N}_{2} \mathrm{H}^{+}\right)\right]$, as a function of mass surface density. Values or $R$ less than 1 indicates chemically evolved material (see text). The observed $R$ drop is consistent with $\mathrm{CO}$ being depleted at high densities due to the freeze-out.

ing toward the denser Filament 2 and causing the observed blueshift between the $\mathrm{C}^{18} \mathrm{O}$ and $\mathrm{N}_{2} \mathrm{H}^{+}$lines. The relative velocity of Filaments 1 and 3 along the line of sight is $3 \mathrm{~km} \mathrm{~s}^{-1}$ (Figure 9). Assuming that the velocity components along the plane of the sky have similar magnitudes, this implies a collision velocity of $\sim 5 \mathrm{~km} \mathrm{~s}^{-1}$. The (slow) shock which followed the impact has caused a density increase in the interaction region. The (post-shock) density enhancement has then contributed to the relatively large average density observed across G035.39-00.33 (Figure15). Before the collision, the filaments appear to be quiescent and coherent in velocity, as suggested by the relatively narrow lines and small velocity variations observed in the North (Figure 8), where Filament 3 appears spatially separated from Filament 1 . It is interesting to note that the velocity coherence is maintained after collision, as indicated by the presence of velocity gradients similar to those measured in nearby low-mass dense cores $\left(\simeq 1 \mathrm{~km} \mathrm{~s}^{-1} \mathrm{pc}^{-1}\right.$, see Section 3.2.2).

Note that, in this scenario, shock velocities of about $5 \mathrm{~km} \mathrm{~s}^{-1}$ in gas of $\simeq 10^{3} \mathrm{~cm}^{-3}$ appear sufficient to inject enough silicon in the gas phase to allow $\mathrm{SiO}$ to form and be detected across G035.39-00.33 (Paper I). However, these values of shock velocities and densities are too low for sputtering and grain-grain collisions in magnetized shocks to be effective in releasing silicon into the gas phase (e.g. Caselli et al. 1997, Jiménez-Serra et al. 2008, Gusdorf et al. 2008, Guillet et al. 2011). It has been discussed in Paper I, that in order to reproduce the observed fractional abundance of $\mathrm{SiO}$ via sputtering within C-type shocks, some $\mathrm{SiO}$ has to be present in the icy mantles (see also Schilke et al. 1997 and Jimenez-Serra et al. 2008), which need a shock velocity slightly above $10 \mathrm{~km} \mathrm{~s}^{-1}$ to be sputtered. Therefore if sputtering is indeed the mechanism producing the observed 


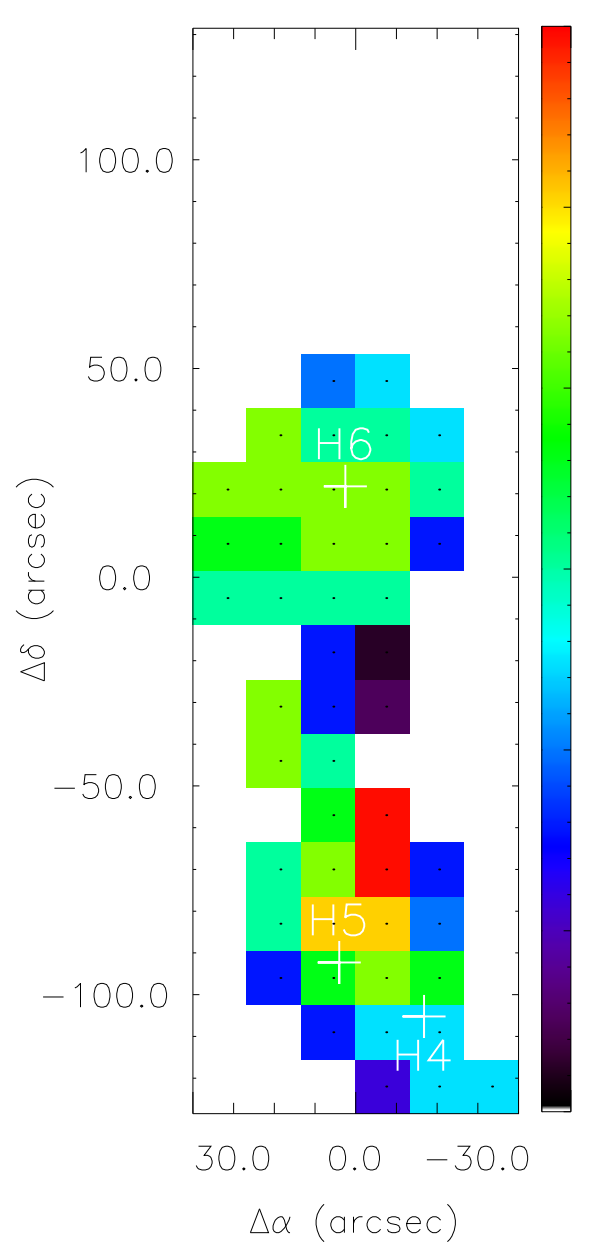

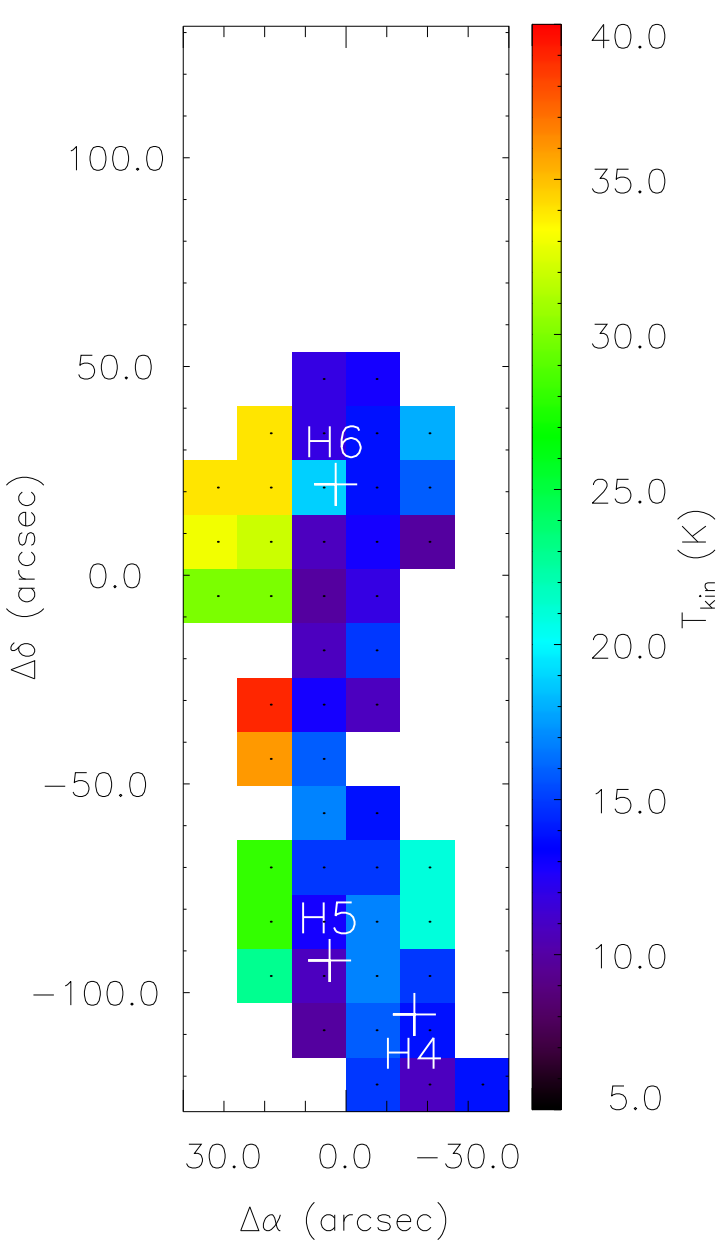

Figure 15. Number density map of G035.39-00.33 obtained from the observed $\mathrm{N}_{2} \mathrm{H}^{+}(3-2) /(1-0)$ line intensity ratio and the use of the RADEX code. A constant kinetic temperature of $15 \mathrm{~K}$ is assumed across the cloud. Some regions at the edge of the filament show significantly higher density values than the average value, suggesting that the assumption of constant temperature may not be valid (see Figure 16). Crosses indicate the position of massive cores from BT12.

$\mathrm{SiO}$ emission, the total velocity in the plane of the sky would have to be at least $10 \mathrm{~km} \mathrm{~s}^{-1}$. Similarly, in case of grain-grain collisions within C-type shocks, the shock velocity has to be at least $10 \mathrm{~km} \mathrm{~s}^{-1}$ to allow dust grains with different electrical charges to achieve a relative velocity larger than the threshold velocity for icy mantle vaporization $\left(6.5 \mathrm{~km} \mathrm{~s}^{-1}\right.$, Tielens et al. 1994; Caselli et al. 1997).

Alternatively, the release of $\mathrm{SiO}$ molecules mixed in icy mantles could be the result of grain mantle vaporization due to grain-grain collisions during the process of filament merging, without invoking C-type shocks. In this scenario, the grain-grain collision velocity can be assumed close to the relative velocities of the merging filaments, which is very close to the threshold velocity mentioned above, especially taking into account the uncertainties in both the observed relative velocity and the theoretical threshold velocity value. In fact, on the one hand, the inferred relative velocity of $\sim 5 \mathrm{~km} \mathrm{~s}^{-1}$

Figure 16. Kinetic temperature map of G035.39-00.33 obtained from the observed $\mathrm{N}_{2} \mathrm{H}^{+}(3-2) /(1-0)$ line intensity ratio and the use of the RADEX code. A constant number density of $5 \times 10^{4} \mathrm{~cm}^{-3}$ (the average number density in Figure 15) is assumed across the cloud. The line intensity ratio at the edge of the dense filament (Filament 2) is consistent with gas temperatures close to $20 \mathrm{~K}$. Crosses indicate the position of massive cores from $\mathrm{BT} 12$.

has been obtained assuming that the velocity components along the plane of the sky have the same magnitude as the component along the line of sight. On the other hand, the $6.5 \mathrm{~km} \mathrm{~s}^{-1}$ value given in Tielens et al. (1994) refers to water ice, with a binding energy larger than $5000 \mathrm{~K}$. If icy mantles are instead made out of a mixture of $\mathrm{H}_{2} \mathrm{O}$ and $\mathrm{CO}$ (as expected in regions with a gas phase dominated by $\mathrm{CO}$ molecules), binding energy may be significantly lower than $5000 \mathrm{~K}$ and the vaporization threshold velocity will be consequently lower. A factor of 5 decrease in binding energy (appropriate for CO molecules bound on an water ice mantle; Öberg et al. 2005) results in about a factor of 3 decrease in the threshold velocity (using the formalism in Tielens et al. 1994). Thus, dust grains moving at relative velocities of about $5 \mathrm{~km} \mathrm{~s}^{-1}$ may lose a significant fraction of their icy mantles upon collision. A way to test this scenario is to observe other species expected to be abundant in the mantles 
of CO-rich dust grains, in particular $\mathrm{CH}_{3} \mathrm{OH}$, which we expect to be widespread across the cloud.

\subsection{The Dynamical Evolution of G035.39-00.33}

The high-sensitivity and high-spectral resolution maps in both $\mathrm{N}_{2} \mathrm{H}^{+}(1-0)$ and $\mathrm{C}^{18} \mathrm{O}(1-0)$ have revealed that the dense gas of the G035.39-00.33 is surrounded by complex, lower density filamentary structures moving at relative velocities of a few $\mathrm{kms}^{-1}$. Multiple velocity components have also been detected in other IRDCs. For instance Devine et al. (2011) found evidence for multiple components separated by similar velocities in IRDC G19.30+0.07. In the previous sections we have shown that in G035.39-00.33, these multiple filaments are interacting.

Given this complexity of the IRDC, the kinematics are relatively quiescent. Toward the northern portion of the IRDC, where star formation activity is at a minimum, the line widths are only a few times the thermal width for a $15 \mathrm{~K}$ gas. Here, $\mathrm{C}^{18} \mathrm{O}(1-0)$ line widths become close to $0.5 \mathrm{~km} \mathrm{~s}^{-1}$, similar to those measured in low mass starforming regions (e.g. Fuller \& Mvers 1992). In the rest of the filament, the lines become a factor of 2-4 broader, probably due to a combination of filament merging and embedded (but not yet prominent) star formation activity (see also Devine et al. 2011, Ragan et al. 2012). Moreover, the velocity structure appears coherent, except toward core H6, where a $1 \mathrm{~km} \mathrm{~s}^{-1} \mathrm{pc}^{-1}$ velocity gradient is measured (probably linked to the adjacent zone of interaction). Figure 7 shows no large velocity gradients throughout the cloud. Hence, the merging of the filaments must be fairly "gentle", possibly regulated by large-scale magnetic fields. The presence of the velocity shift between $\mathrm{C}^{18} \mathrm{O}$ and $\mathrm{N}_{2} \mathrm{H}^{+}$lines is telling us that the accumulation of material toward the region of interaction of the filaments is still ongoing. Assuming a simplified 1D geometry, the time to build up the mass of Filament 2 from gas flowing in from Filaments 1 and 3 at relative velocity $v_{\text {flow }}$ is:

$$
\begin{array}{r}
t_{\text {flow }} \sim 2 \frac{R_{f}}{v_{\text {flow }}} \frac{n_{\mathrm{H}, \mathrm{f}}}{n_{\mathrm{H}, \text { flow }}} \rightarrow \\
2.0\left(\frac{R_{f}}{0.5 \mathrm{pc}}\right)\left(\frac{v_{\text {flow }}}{5 \mathrm{~km} \mathrm{~s}^{-1}}\right)^{-1}\left(\frac{n_{\mathrm{H}, \mathrm{f}} / n_{\mathrm{H}, \text { flow }}}{10}\right) \mathrm{Myr}
\end{array}
$$

where $R_{f}$ is the radius of the filament, here normalized to 0.5 pc, i.e. $36^{\prime \prime}$ (see e.g. left panel of Fig.2), $n_{\mathrm{H}, f}$ is the mean total hydrogen nuclei number density in Filament 2 and $n_{\mathrm{H} \text {,flow }}$ is the mean density in the merging regions of Filaments 1 and 3 . The ratio of these mean densities is difficult to estimate, especially given the possibility of unresolved clumping of the emission of particular gas tracers. From the extinction map, Paper III estimated a density contrast of a factor of 3 for the case of the "Inner Filament" and its immediate envelope, which is likely to be a lower limit to the ratio used in Equation 4.2 Jimenez-Serra et al. (in prep.) find a density contrast of a factor of $\sim 10$, based on $\mathrm{CO}$ excitation analysis. The timescale of $\gtrsim 1$ Myr that is implied for the build-up of Filament 2 by the converging flows of Filaments 1 and 3 is longer than the local dynamical time in Filament $2(\simeq 0.8 \mathrm{Myr})$, a necessary requirement if it has reached virial equilibrium as concluded in Paper III.

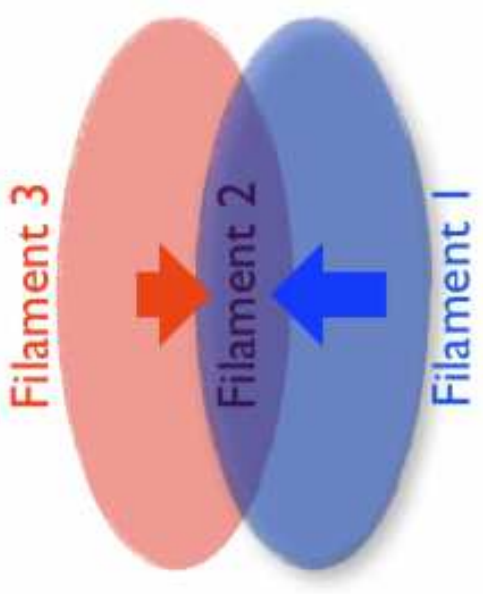

Figure 17. Schematic figure illustrating the kinematics of G035.39-00.33.

We have proposed that the merging of filaments has led to the local density increase along G035.39-00.33 and, in particular at the position of core H6. Cluster formation due to the collision of filaments has been suggested in other star-forming regions, for instance in the massive starforming region W33A (Galván-Madrid et al. 2010), in the low-mass star-forming region Serpens (Duarte-Cabral et al. 2011), in the L1641-N region (Nakamura et al. 2012), and in the Rosette Nebula (Schneider et al. 2012). Here, we have been able, using high spectral, high sensitivity data, to show that in this particular IRDC, the merging of filaments appears to be occurring gently, at a relatively low velocity, and that the build-up of material may go on for several million years.

\section{SUMMARY \& CONCLUSIONS}

We have taken high sensitivity and high spectral resolution images of the IRDC G035.39-00.33, chosen because of the cloud's characteristics suggesting it is in an early stage of its evolution. From our analysis we have found the following:

i) Rather than just the single prominent filament that is seen in extinction, we find two additional, morphologicallydistinct, filaments with velocity components separated by a few separated by a few $\mathrm{km} \mathrm{s}^{-1}$. In Filament 2, we find that $\mathrm{N}_{2} \mathrm{H}^{+}(1-0)$ traces the whole filament and not just the cores. There is a local maximum at the position of core H6, and this massive core is spatially coincident with the point of intersection of the velocity components. We suggest that the merging of Filaments 1 and 3 have produced the IRDC (Filament 2)..

ii) Our kinematic study has revealed large-scale velocity coherence (comparable line widths at most positions, lack of large velocity gradients etc.) across the whole cloud. This is indicative of a "gentle" merging of filaments. We suggest that the merging of the filaments in the ongoing cloud formation process may be responsible for the large-scale SiO $(2-1)$ emission seen in the Jiménez-Serra et al. (2010) study.

iii) We have also witnessed for the first time, a 
widespread velocity shift $\left(\sim 0.2 \mathrm{~km} \mathrm{~s}^{-1}\right)$ between the $\mathrm{N}_{2} \mathrm{H}^{+}$ $(1-0)$ and $\mathrm{C}^{18} \mathrm{O}(1-0)$. Conversely, in low-mass regions, no velocity shift is seen between these two tracers. The presence of a velocity shift between these tracers over large scales is consistent with ongoing merging of filaments, which started $\gtrsim 1$ Myr ago. Star formation within this cloud may have been locally induced by the IRDC formation process (filament merging / cloud-could collision).

iv) Analysis using radiative transfer code, RADEX, gives an average $\mathrm{H}_{2}$ number density across the IRDC of about $5 \times 10^{4} \mathrm{~cm}^{-3}$ and there is indication of a temperature gradient perpendicular to the filament.

v) We find similarities and differences between the studied IRDC and nearby (more quiescent) star-forming regions. Among the similarities we list: (1) material that exhibits quiescent kinematics, i.e. coherent velocities, especially in the northern regions where star formation activity is at a minimum (suggesting that the filaments have initially physical properties similar to those found in lowmass star-forming regions); (2) small velocity gradients $\left(\simeq 1 \mathrm{~km} \mathrm{~s}^{-1} \mathrm{pc}^{-1}\right)$; (3) fractional abundances with respect to $\mathrm{H}_{2}$ molecules of $\mathrm{N}_{2} \mathrm{H}^{+} \simeq 4 \times 10^{-10}$. Among the differences we found: (1) non-thermally dominated motions traced by $\mathrm{N}_{2} \mathrm{H}^{+}$, perhaps due to the fact that the dense gas in the IRDC has been and continues to be formed from the collision of supersonically interacting filaments; (2) large-scale emission of $\mathrm{N}_{2} \mathrm{H}^{+}(1-0)$ and $(3-2)$, implying relatively large densities $\left(n_{\mathrm{H}} \sim 10^{5} \mathrm{~cm}^{-3}\right)$ extend over large, parsecscale regions in the IRDC.

vi) This study has also shown the importance of high spectral resolution of different gas tracers to unveil the kinematics and correctly interpret line asymmetries. Asymmetric blue-peaked profiles found across G035.39-00.33 are due to multiple velocity components along the line of sight. We did not find any evidence of large-scale infall motions. Instead, we find evidence of ongoing accumulation of material at the intersection of two merging of filaments. Higher angular resolution observations are needed to isolate single centers of accretion (i.e. the star-forming dense cores analogous to the well-studied low-mass cores).

\section{ACKNOWLEDGEMENTS}

We acknowledge the IRAM staff for the help provided during the observations. We thank Michael Butler and Jouni Kainulainen for providing the extinction maps for this work. We thank Jaime E. Pineda for assistance with image processing. PC work on star formation is supported by a STFC rolling grant.

\section{REFERENCES}

André P., Belloche A., Motte F., Peretto N., 2007, A\&A, 472,519

Butler M. J., Tan J. C., 2009, ApJ, 696, 484

Butler M. J., Tan J. C., 2012, ApJ, 754, 5

Carey S. J., Clark F. O., Egan M. P., Price S. D., Shipman

R. F., Kuchar T. A., 1998, ApJ, 508, 721

Caselli P., Benson P. J., Myers P. C., Tafalla M., 2002a, ApJ, 572, 238
Caselli P., Hartquist T. W., Havnes O., 1997, A\&A, 322, 296

Caselli P., Myers P. C., Thaddeus P., 1995, ApJL, 455, L77+

Caselli P., Walmsley C. M., Tafalla M., Dore L., Myers P. C., 1999, ApJL, 523, L165

Caselli P., Walmsley C. M., Zucconi A., Tafalla M., Dore L., Myers P. C., 2002b, ApJ, 565, 344

Cazzoli G., Puzzarini C., Lapinov A. V., 2003, ApJL, 592, L95

Chen H.-R., Liu S.-Y., Su Y.-N., Zhang Q., 2010, ApJL, 713, L50

Crapsi A. et al., 2005, A\&A, 439, 1023

Devine K. E., Chandler C. J., Brogan C., Churchwell E., Indebetouw R., Shirley Y., Borg K. J., 2011, ApJ, 733, 44

Duarte-Cabral A., Dobbs C. L., Peretto N., Fuller G. A., 2011, A\&A, 528, A50

Egan M. P., Shipman R. F., Price S. D., Carey S. J., Clark F. O., Cohen M., 1998, ApJL, 494, L199+

Fontani F. et al., 2011, A\&A, 529, L7+

Friesen R. K., Di Francesco J., Shimajiri Y., Takakuwa S., 2010, ApJ, 708, 1002

Fuller G. A., Myers P. C., 1992, ApJ, 384, 523

Galván-Madrid R., Zhang Q., Keto E., Ho P. T. P., Zapata L. A., Rodríguez L. F., Pineda J. E., Vázquez-Semadeni E., 2010, ApJ, 725, 17

Guillet V., Pineau Des Forêts G., Jones A. P., 2011, A\&A, 527, A123

Gusdorf A., Cabrit S., Flower D. R., Pineau Des Forêts G., 2008, A\&A, 482, 809

Hacar A., Tafalla M., 2011, A\&A, 533, A34

Heitsch F., Stone J. M., Hartmann L. W., 2009, ApJ, 695, 248

Hennebelle P., Banerjee R., Vázquez-Semadeni E., Klessen R. S., Audit E., 2008, A\&A, 486, L43

Hernandez A. K., Tan J. C., 2011, ApJ, 730, 44

Hernandez A. K., Tan J. C., Caselli P., Butler M. J., Jiménez-Serra I., Fontani F., Barnes P., 2011, ApJ, 738, 11, (Paper II)

Hernandez A. K., Tan J. C., Kainulainen J., Caselli P., Butler M. J., Jiménez-Serra I., Fontani F., 2012, ApJL, 756, L13, (Paper III)

Hily-Blant P., Walmsley M., Pineau Des Forêts G., Flower D., 2010, A\&A, 513, A41+

Jiménez-Serra I., Caselli P., Martín-Pintado J., Hartquist T. W., 2008, A\&A, 482, 549

Jiménez-Serra I., Caselli P., Tan J. C., Hernandez A. K., Fontani F., Butler M. J., van Loo S., 2010, MNRAS, 406, 187, (Paper I)

Jiménez-Serra I., Martín-Pintado J., Caselli P., Viti S., Rodríguez-Franco A., 2009, ApJ, 695, 149

Kainulainen J., Tan J. C., 2012. ApJ, Submitted

Kirk H., Johnstone D., Tafalla M., 2007, ApJ, 668, 1042

Kutner M. L., Ulich B. L., 1981, ApJ, 250, 341

Miettinen O., Harju J., Haikala L. K., Juvela M., 2012, A\&A, 538, A137

Miettinen O., Hennemann M., Linz H., 2011, A\&A, 534, A134

Mizuno A., Onishi T., Yonekura Y., Nagahama T., Ogawa H., Fukui Y., 1995, ApJL, 445, L161

Nakamura F. et al., 2012, ApJ, 746, 25

Nguyen Luong Q. et al., 2011, A\&A, 535, A76 
Henshaw, Caselli, Fontani, Jiménez-Serra, Tan, Hernandez

Pagani L., Bacmann A., Cabrit S., Vastel C., 2007, A\&A, 467, 179

Pagani L., Daniel F., Dubernet M., 2009, A\&A, 494, 719

Perault M. et al., 1996, A\&A, 315, L165

Peretto N. et al., 2010, A\&A, 518, L98

Pillai T., Wyrowski F., Carey S. J., Menten K. M., 2006, A\&A, 450, 569

Pillai T., Wyrowski F., Hatchell J., Gibb A. G., Thompson M. A., 2007, A\&A, 467, 207

Ragan S. E., Bergin E. A., Wilner D., 2011, ApJ, 736, 163

Ragan S. E., Heitsch F., Bergin E. A., Wilner D., 2012, ApJ, 746, 174

Rathborne J. M., Jackson J. M., Simon R., 2006, ApJ, 641, 389

Schneider N. et al., 2012, A\&A, 540, L11

Simon R., Rathborne J. M., Shah R. Y., Jackson J. M., Chambers E. T., 2006, ApJ, 653, 1325

Tafalla M., Myers P. C., Caselli P., Walmsley C. M., Comito C., 2002, ApJ, 569, 815

Tafalla M., Santiago J., 2004, A\&A, 414, L53

van der Tak F. F. S., Black J. H., Schöier F. L., Jansen

D. J., van Dishoeck E. F., 2007, A\&A, 468, 627

van Loo S., Falle S. A. E. G., Hartquist T. W., Moore T. J. T., 2007, A\&A, 471, 213

Vasyunina T., Linz H., Henning T., Stecklum B., Klose S., Nyman L.-A., 2009, A\&A, 499, 149

Vasyunina T., Linz H., Henning T., Zinchenko I., Beuther H., Voronkov M., 2011, A\&A, 527, A88

Walsh A. J., Myers P. C., Burton M. G., 2004, ApJ, 614, 194

\section{APPENDIX A: THE GUIDED GAUSSIAN FIT}

Given the complexity of the data, we have developed a semiautomatic procedure in order to fit and interpret the data, dubbed the Guided Gaussian Fit (GGF). The fitting method is based on defining multiple velocity components of gas along the line of sight. In the case of G035.39-00.33, we observe three velocity components of differing morphologies (see Section 3.2.1 and Figures 5 \& 6). Based on the emission seen in Figure 6] we define three windows in CLASS: $42-44 \mathrm{~km} \mathrm{~s}^{-1}, 44-46 \mathrm{~km} \mathrm{~s}^{-1}$, and $46-48 \mathrm{~km} \mathrm{~s}^{-1}$. Using these three windows as a guide, we then fit three Gaussian profiles to the average spectrum of both species. The method here for the $\mathrm{C}^{18} \mathrm{O}(1-0)$ is straightforward in that the three components are seen at numerous positions throughout the cloud and therefore are evident in the average spectrum. This result can be seen in Figure A1 Here, the average spectrum of $\mathrm{C}^{18} \mathrm{O}(1-0)$ is displayed with three Gaussian profiles overlaid. These peak at velocities of, (Filament 1) $43.411 \mathrm{~km} \mathrm{~s}^{-1}$, (Filament 2) $45.197 \mathrm{~km} \mathrm{~s}^{-1}$, and (Filament 3) $46.330 \mathrm{~km} \mathrm{~s}^{-1}$. In the $\mathrm{N}_{2} \mathrm{H}^{+}(1-0)$, Filaments 1 and 3 are only seen at a relatively small number of positions (compared to Filament 2). Thus, for the Filament 1 and 3 components of $\mathrm{N}_{2} \mathrm{H}^{+}$we take the average spectrum over those positions where these components are detected, rather than over the full cloud, to avoid dilution. Centroid velocities, as determined by the GGF, for $\mathrm{N}_{2} \mathrm{H}^{+}(1-0)$ of Filaments 1,2 , and 3 are $42.985 \mathrm{~km} \mathrm{~s}^{-1}, 45.582 \mathrm{~km} \mathrm{~s}^{-1}$, and $46.834 \mathrm{~km} \mathrm{~s}^{-1}$, respectively.

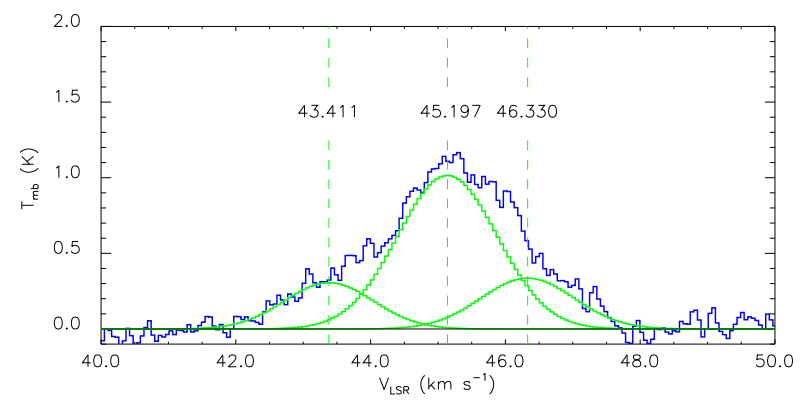

Figure A1. Average spectra of the $\mathrm{C}^{18} \mathrm{O}(1-0)$ shown in blue. Overlaid in green are the three components associated with the average spectrum at velocities, $43.411 \mathrm{~km} \mathrm{~s}^{-1}, 45.197 \mathrm{~km} \mathrm{~s}^{-1}$, and $46.330 \mathrm{~km} \mathrm{~s}^{-1}$.

By taking the line width output from the resulting fit, we can calculate the velocity dispersion using,

$$
\sigma_{V L S R}=\frac{\Delta v}{2 \sqrt{2 \ln (2)}}
$$

We can now use this as the velocity window within which we search for the three velocity components. CLASS is then used to calculate the integrated intensity over these three windows. The standard detection threshold for Gaussian profiles is the $3 \sigma$ level. In the case of G035.39-00.33, we see three typically overlapping components along the line of sight. We have therefore held the detection limit for any individual component at $\mathrm{S} / \mathrm{N} \geqslant 9$.

Using this as a guide we now know for any given spectra (in $\mathrm{C}^{18} \mathrm{O}(1-0)$ ), whether 1,2 or 3 Gaussians should be fitted, and which filaments should be fitted. In order to further verify this, the less complicated isolated component of $\mathrm{N}_{2} \mathrm{H}^{+}$ $(1-0)$ is overlaid on the spectra of the $\mathrm{C}^{18} \mathrm{O}$. Approximately, at any given position where $\mathrm{N}_{2} \mathrm{H}^{+}(1-0)$ is observed one can also expect to see $\mathrm{C}^{18} \mathrm{O}(1-0)$. Therefore, our "extraguide" to our kinematic study is the $\mathrm{N}_{2} \mathrm{H}^{+}(1-0)$ emission. If mutliple components are detected in $\mathrm{N}_{2} \mathrm{H}^{+}(1-0)$, and this is reflected in the spectra of the $\mathrm{C}^{18} \mathrm{O}$, then the same components are recorded in both species. If a component detected in $\mathrm{C}^{18} \mathrm{O}(1-0)$ is not detected in $\mathrm{N}_{2} \mathrm{H}^{+}(1-0)$, then the $\mathrm{C}^{18} \mathrm{O}(1-0)$ emission must be checked using the GGF intensity and the gas distribution seen in Figure 6] If indeed the feature meets these two conditions, it is deduced to be a component in its own right and is fitted as such. However, if the feature is indistinguishable as a component in its own right, either due to blending of two filaments, or perhaps depletion features, it is flagged as a blended line, and is removed from the analysis. An example of this fitting proceedure can be seen in Figure A2, where by we plot the spectra of $\mathrm{C}^{18} \mathrm{O}(1-0)$ in blue, and $\mathrm{N}_{2} \mathrm{H}^{+}(1-0)$ in red, at offset $(-7.5,21)$, at approximately the position of core $\mathrm{H} 6$. The results of the guided Gaussian fit are shown in green and yellow, respectively.

\section{APPENDIX B: GAUSSIAN AND HFS FIT RESULTS IN SELECTED POSITIONS}




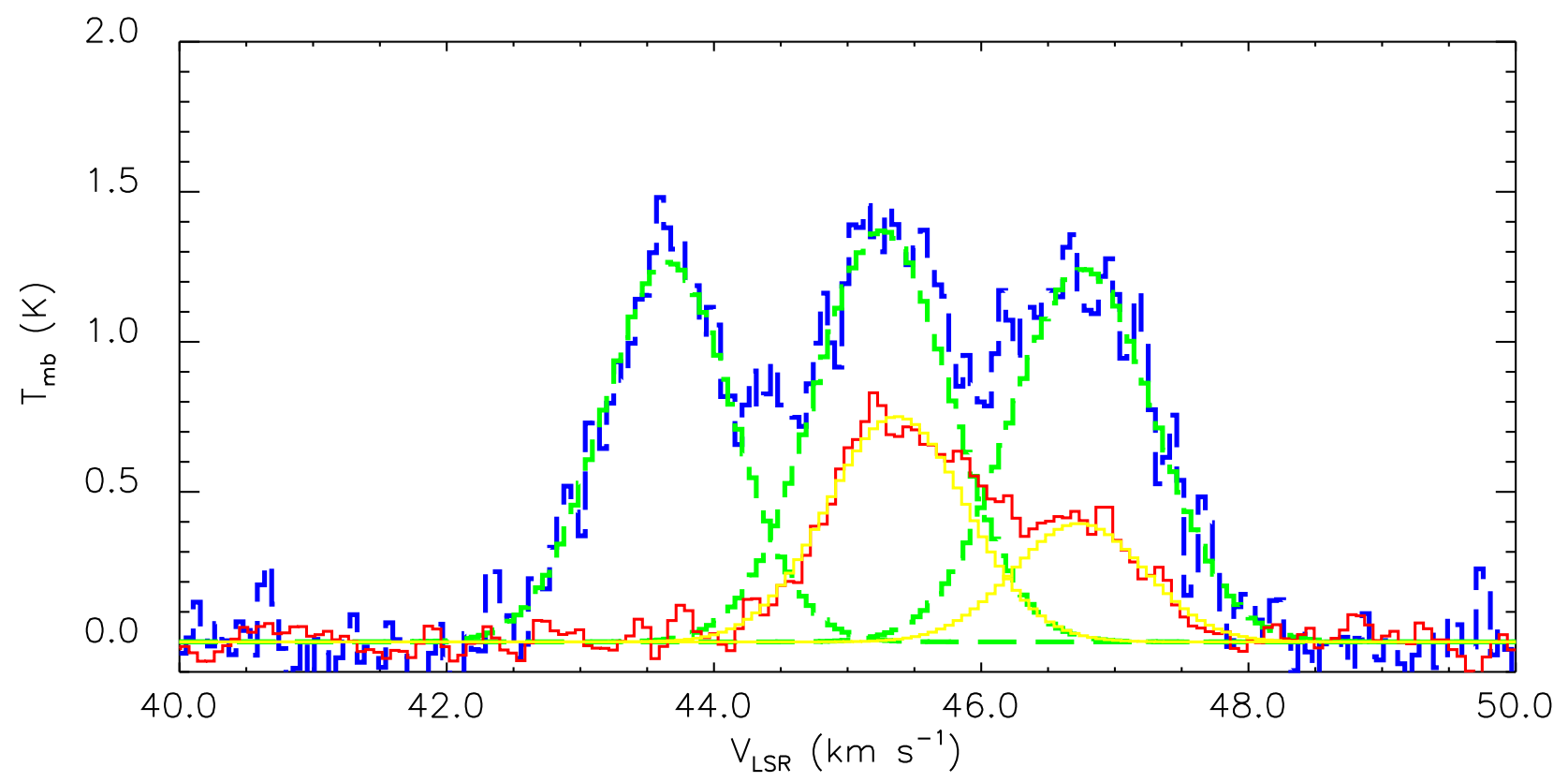

Figure A2. Spectra at offset $(-7.5,21)$. $\mathrm{C}^{18} \mathrm{O}(1-0)$ is shown as the dashed profile, in blue with the results of the GGF shown in green. $\mathrm{N}_{2} \mathrm{H}^{+}(1-0)$ is shown as the solid profile, in red with the results of the GGF in yellow.

Table B1. Results of the guided Gaussian fitting method at selected offsets.

\begin{tabular}{|c|c|c|c|c|c|}
\hline Position & $\overline{\overline{\text { Filament }}}$ & $\overline{\mathrm{T}_{m b}(\mathrm{~K})}$ & $\overline{\mathrm{II}\left(\mathrm{K} \mathrm{km} \mathrm{s}^{-1}\right)}$ & $\overline{\mathrm{V}_{L S R}\left(\mathrm{~km} \mathrm{~s}^{-1}\right)}$ & $\overline{\Delta \Delta v\left(\mathrm{~km} \mathrm{~s}^{-1}\right)}$ \\
\hline \multicolumn{6}{|c|}{$\mathrm{N}_{2} \mathrm{H}^{+}(1-0)$} \\
\hline \multirow[t]{2}{*}{$(-7.5,21.0)$} & Filament 2 & $0.75(0.04)$ & $0.98(0.06)$ & $45.37(0.03)$ & $1.23(0.06)$ \\
\hline & Filament 3 & $0.40(0.04)$ & $0.49(0.05)$ & $46.73(0.06)$ & $1.16(0.11)$ \\
\hline \multirow[t]{2}{*}{$(5.5,21.0)$} & Filament 2 & $0.93(0.04)$ & $1.11(0.03)$ & $45.32(0.01)$ & $1.12(0.02)$ \\
\hline & Filament 3 & $0.38(0.04)$ & $0.61(0.02)$ & $46.52(0.02)$ & $1.52(0.06)$ \\
\hline$(5.5,-31.0)$ & Filament 2 & $0.58(0.04)$ & $0.78(0.02)$ & $45.53(0.01)$ & $1.26(0.03)$ \\
\hline$(5.5,-83.0)$ & Filament 1 & $0.80(0.03)$ & $1.10(0.02)$ & $45.35(0.01)$ & $1.29(0.02)$ \\
\hline \multicolumn{6}{|c|}{$\mathrm{C}^{18} \mathrm{O}(1-0)$} \\
\hline \multirow[t]{3}{*}{$(-7.5,21.0)$} & Filament 1 & $1.27(0.11)$ & $1.55(0.07)$ & $43.66(0.02)$ & $1.15(0.05)$ \\
\hline & Filament 2 & $1.37(0.11)$ & $1.75(0.12)$ & $45.26(0.02)$ & $1.20(0.08)$ \\
\hline & Filament 3 & $1.24(0.11)$ & $1.67(0.09)$ & $46.76(0.03)$ & $1.27(0.07)$ \\
\hline \multirow[t]{3}{*}{$(5.5,21.0)$} & Filament 1 & $0.78(0.10)$ & $1.04(0.05)$ & $43.59(0.03)$ & $1.25(0.07)$ \\
\hline & Filament 2 & $1.99(0.10)$ & $2.37(0.10)$ & $45.29(0.02)$ & $1.12(0.04)$ \\
\hline & Filament 3 & $1.13(0.10)$ & $1.47(0.08)$ & $46.72(0.03)$ & $1.23(0.07)$ \\
\hline$(5.5,-31.0)$ & Filament 2 & $1.65(0.13)$ & $3.46(0.06)$ & $45.55(0.02)$ & $1.97(0.04)$ \\
\hline$(5.5,-83.0)$ & Filament 2 & $2.01(0.14)$ & $3.45(0.06)$ & $45.13(0.01)$ & $1.61(0.04)$ \\
\hline
\end{tabular}


Table B2. Results of the HFS fitting method at selected offsets.

\begin{tabular}{cccccc}
\hline \hline Position & $\mathrm{T}_{a n t}{ }^{* T a u}(\mathrm{~K})$ & $\mathrm{V}_{L S R}\left(\mathrm{~km} \mathrm{~s}^{-1}\right)$ & $\Delta v\left(\mathrm{~km} \mathrm{~s}^{-1}\right)$ & $\tau$ & $\mathrm{T}_{e x}$ \\
\hline \multicolumn{7}{c}{$\mathrm{N}_{2} \mathrm{H}^{+}(1-0)$} \\
$(-7.5,21.0)$ & $7.01(0.13)$ & $45.74(0.01)$ & $1.94(0.02)$ & $3.48(0.12)$ & $5.0(0.1)$ \\
$(5.5,21.0)$ & $9.06(0.13)$ & $45.63(0.01)$ & $1.70(0.01)$ & $3.42(0.10)$ & $5.6(0.1)$ \\
$(5.5,-31.0)$ & $5.14(0.17)$ & $45.48(0.01)$ & $1.40(0.02)$ & $3.07(0.23)$ & $4.6(0.2)$ \\
\hline \multicolumn{7}{c}{$-83.0)$} & $7.70(0.15)$ & $45.38(0.01)$ & $1.41(0.01)$ & $4.28(0.16)$ & $4.7(0.1)$ \\
\hline$(-4.0,23.0)$ & $0.99(0.06)$ & $45.83(0.08)$ & $2.40(0.19)$ & 0.10 & $\cdots$ \\
$(9.0,23.0)$ & $1.41(0.10)$ & $45.43(0.05)$ & $1.65(0.16)$ & 0.10 & $\cdots$ \\
$(9.0,-29.0)$ & $0.61(0.09)$ & $45.69(0.09)$ & $0.81(0.30)$ & 0.10 & $\cdots$ \\
$(9.0,-81.0)$ & $0.85(0.07)$ & $45.36(0.09)$ & $2.02(0.20)$ & 0.10 & $\cdots$ \\
\hline \hline
\end{tabular}

